OPEN ACCESS

Edited by: Gianluca Reali,

University of Perugia, Italy

Reviewed by:

Pasquale Stano,

University of Salento, Italy

Valeria Loscri,

Inria research centre Lille - Nord

Europe, France

${ }^{*}$ Correspondence:

Meltem Civas

mcivas16@ku.edu.tr

Oktay Cetinkaya

ocetinkaya@ku.edu.tr

Murat Kuscu

mkuscu@ku.edu.tr

Specialty section:

This article was submitted to Non-Conventional Communications and Networks,

a section of the journal Frontiers in Communications and Networks

Received: 30 June 2021 Accepted: 16 September 2021 Published: 29 September 2021

Citation:

Civas M, Cetinkaya O, Kuscu M and Akan OB (2021) Universal

Transceivers: Opportunities and Future Directions for the Internet of Everything (IOE).

Front. Comms. Net 2:733664. doi: 10.3389/frcmn.2021.733664

\section{Universal Transceivers: Opportunities and Future Directions for the Internet of Everything (IoE)}

\author{
Meltem Civas ${ }^{1 *}$, Oktay Cetinkaya ${ }^{1 *}$, Murat Kuscu $^{1 *}$ and Ozgur B. Akan ${ }^{1,2}$ \\ ${ }^{1}$ The Department of Electrical and Electronics Engineering, Koç University, Istanbul, Turkey, ${ }^{2}$ Internet of Everything (loE) Group, \\ Electrical Engineering Division, Department of Engineering, University of Cambridge, Cambridge, United Kingdom
}

The Internet of Everything (loE) is a recently introduced information and communication technology (ICT) framework promising for extending the human connectivity to the entire universe, which itself can be regarded as a natural loE, an interconnected network of everything we perceive. The countless number of opportunities that can be enabled by loE through a blend of heterogeneous ICT technologies across different scales and environments and a seamless interface with the natural loE impose several fundamental challenges, such as interoperability, ubiquitous connectivity, energy efficiency, and miniaturization. The key to address these challenges is to advance our communication technology to match the multi-scale, multi-modal, and dynamic features of the natural loE. To this end, we introduce a new communication device concept, namely the universal $\mathrm{loE}$ transceiver, that encompasses transceiver architectures that are characterized by multi-modality in communication (with modalities such as molecular, $\mathrm{RF} / \mathrm{THz}$, optical and acoustic) and in energy harvesting (with modalities such as mechanical, solar, biochemical), modularity, tunability, and scalability. Focusing on these fundamental traits, we provide an overview of the opportunities that can be opened up by micro/nanoscale universal transceiver architectures towards realizing the loE applications. We also discuss the most pressing challenges in implementing such transceivers and briefly review the open research directions. Our discussion is particularly focused on the opportunities and challenges pertaining to the loE physical layer, which can enable the efficient and effective design of higher-level techniques. We believe that such universal transceivers can pave the way for seamless connection and communication with the universe at a deeper level and pioneer the construction of the forthcoming loE landscape.

Index Terms- Internet of Everything, Universal loE Transceiver, Interoperability, Multimodality, Hybrid Energy Harvesting, Molecular Communications, $\mathrm{THz}$ Communications, Graphene and related nanomaterials.

Keywords: universal IoE transceiver, interoperability, multi-modality, hybrid energy harvesting, molecular communications, $\mathrm{THz}$ communications, graphene and related nanomaterials, internet of everything 


\section{INTRODUCTION}

The ever-growing demand for high data rates and low latency coupled with the exponential increase in the number of interconnected devices has been met by the evolution and diversification of the conventional communication technologies, particularly moving towards higher frequency bands, e.g., mm-Wave and $\mathrm{THz}$ bands (Akyildiz et al., 2020). Likewise, the objective of extending our control and connectivity to underexplored environments, e.g., underwater, intra-body, with the ever-increasing resolution has led to the emergence of new non-conventional communication modalities, such as acoustic communications and molecular communications (MC) (Akyildiz et al., 2019; Song et al., 2019). These fastpaced developments have been further accompanied by the introduction of numerous wireless power transfer (WPT) and energy harvesting $(\mathrm{EH})$ techniques that can exploit various energy sources to overcome the limitations of finite-capacity batteries and enable self-sustaining networks within the Internet of Things (IoT) framework (Akan et al., 2018; Kamalinejad et al., 2015). Hence, the current and future landscapes of communications and networking are characterized by increasing heterogeneity of communication and power supply technologies, which are optimized only for particular scales, environments, and applications.

The recently introduced Internet of Everything (IoE) framework is positioned to exploit the heterogeneity of current and nextgeneration communication and networking technologies (both conventional and non-conventional) to extend our connectivity to the entire universe, which is itself a natural IoE, an inherently heterogeneous network of everything we perceive, whose interactions governed by the laws of physics (Dinc et al., 2019). Maximizing the connectivity to the universal scale through the integration of different communication technologies and their close interaction with the natural $\mathrm{IoE}$ is expected to enable novel applications. For example, control over biological communication pathways among living entities, such as biological cells, animals, plants, through the seamless interface of our communication technologies will have broad implications for biomedical, agricultural, and environmental applications (Akyildiz et al., 2015). As a particular example, interfacing future molecular nanosensor networks located inside the human brain to the low-latency and high-rate electromagnetic $5 \mathrm{G}$ wireless networks can bring the Internet of Senses to reality, enabling the nonverbal, i.e., conceptual, communication of human-body senses between individuals. The countless number of applications that can be enabled by the blend of heterogeneous technologies across different scales and environments, however, impose several fundamental challenges, such as interoperability, ubiquitous connectivity, energy efficiency, and miniaturization.

As in all networking technologies, the opportunities and limitations pertaining to the physical layer of the IoE will largely determine what we can achieve with higher-layer techniques and protocols in terms of tackling its core challenges. With this consideration, we set out to investigate the physical architecture of a universal transceiver that, we believe, can enable the efficient and effective design of higher-level techniques for the IoE. Here we coin the universal IoE transceiver as an umbrella term, which encompasses all transceiver devices that are characterized by the key IoE attributes, such as multi-modality, modularity, tunability, and scalability, and can be embedded into any entity or device to transform them into IoE devices. Although combinations of these traits have already been considered in the literature for various communication devices and networks, those studies are limited to particular communication technologies and applications, and thus, away from providing general insights into transceiver architectures that can address the IoE challenges. For example, multi-modal communications has been extensively studied in the context of underwater networks to optimize the connectivity by adaptively combining acoustic communications with optical or RF communications (Basagni et al., 2019; Campagnaro, 2018; Zhao, 2020). Smart gateway architectures, providing modularity and multi-modality in terms of communication technologies and protocols, have been proposed for IoT to shield the heterogeneity of the IoT sensing layer in its interaction with the network layer (Guoqiang, 2013). Cognitive radio, and in general, software-defined radio technologies consist in the dynamic tunability of the transmission and reception parameters of network nodes, which gives their flexibility in overcoming the limited spectrum challenges (Zalonis and Polydoros, 2005; Arslan, 2007; Cetinkaya et al., 2020a). Multi-modal, bio-cyber, and macronano interface architectures have been proposed for MC, which transduce MC signals to different signal forms including RF and optical (Kuscu and Akan, 2015; Nakano et al., 2014; Koucheryavy, 2021).

Focusing on the fundamental traits introduced above, this paper provides an overview of the opportunities that are made possible by such universal transceiver architectures. That is performed in the context of IoE, such that the overview is not focused on particular technologies or applications. However, example scenarios of practical applications accompany the discussion to justify the need for universal transceivers combining these features. We also discuss the most important challenges in implementing such transceivers and briefly review potential research directions to practically address them. Although our discussion is focused on physical layer challenges, we occasionally extend it to higher layers to provide a broader perspective.

\section{UNIVERSAL IOE TRANSCEIVER}

We envision a universal IoE transceiver as a physical device enabling the design of higher-level communication techniques and protocols that would help overcome the fundamental IoE challenges, such as interoperability, ubiquitous connectivity, energy efficiency, miniaturization, and scarcity of bandwidth. Although this paper is not proposing a particular architecture, the following set of properties delineates the universal IoE transceiver, which is investigated in this paper in terms of opportunities and challenges:

- Multi-modality in communications: The universal IoE transceiver is capable of transceiving communication 
signals in multiple forms, e.g., RF, optical, molecular, and acoustic. Supporting multiple combinations of communication modalities, the universal transceiver will bring important functionalities, such as adaptivity, multiplexing, and seamless interfacing between different modalities as discussed in Section 3, which will help overcome the interoperability and ubiquitous connectivity challenges of the IoE.

- Multi-modality in EH: The considered device has an architecture that can harvest energy from multiple energy sources, such as RF, mechanical, biochemical, and solar energy sources, as well as, utilize WPT in an hybrid manner. The adaptivity enabled by multi-modality in energy supply will effectively address the energy scarcity challenges in the IoE.

- Modularity: The device has a modular architecture that enables reconfigurability. This will allow the utilization of the most appropriate communication and $\mathrm{EH}$ modalities for particular applications and application environments, eliminating any redundancy. Future communication and $\mathrm{EH}$ technologies can also be integrated into the universal transceiver thanks to its modular structure.

- Tunability: The dynamic tunability of transmission and reception parameters for individual communication modalities, e.g., the frequency of transmit signals in RF communications or the type of carrier molecules in MC, will contribute to the adaptivity, energy efficiency, and environment-compatibility of the universal transceiver, as discussed in Section 3.

- Scalability: The device architecture is scalable to micro/ nanoscales, supporting ubiquitous connectivity especially in bio-nano environments and enabling the deployment of more IoE functionalities in smaller volumes.

In the following, we address the PHY/device opportunities and challenges for a universal IoE transceiver defined by aforementioned properties. Extension of the investigation to upper layers (e.g., network layer) within the IoE framework can be considered as a future work.

\section{OPPORTUNITIES OF UNIVERSAL IOE TRANSCEIVERS}

The universal IoE transceivers can present unprecedented opportunities within the emerging IoE framework by supporting the interoperability of heterogeneous communication networks through seamlessly interfacing their physical layers, providing ubiquitous operation and adaptivity at diverse and dynamic environments, and enabling the opportunistic utilization of multiple communication and energy modalities. In this section, we present a detailed discussion of these opportunities along with the potential applications.

\subsection{Heterogeneous IoE}

The current landscape of networking is already characterized by heterogeneity of communication technologies. On top of that, our perennial quest to reach out to underexplored environments, such as underwater and intra-body, is pushing the diversification of the communication technologies to new levels. Besides, natural communication systems, which are optimized through billions of years of evolution, are also characterized by substantial heterogeneity, for example, in terms of biological signalling pathways and signalling entities.

IoE, which aims at integrating these conventional and unconventional communication mechanisms involved in these heterogeneous networks to merge natural and human-made networks and maximize our connectivity to the universal scale, poses a great interoperability challenge.

Nature has developed its unique and elegant ways of overcoming the interoperability challenge in interconnecting different species through inter-kingdom communication pathways which lend itself to intriguing consequences that are yet to be fully uncovered. For example, the signalling through gutbrain axis connects the nerve cells in human central nervous system and the microbes in the gastrointestinal tract, which have implications in many human-body disorders and diseases, such as depression and irritable bowel syndrome (Foster and McVey Neufeld, 2013). Plants and animals communicate with diverse signalling mechanisms, e.g., visual and biochemical signals released by the plants attract the animals that can disperse the seeds of the plant, or, bat-pollinated plants can communicate with the bats via acoustic signals (Schöner et al., 2016). Similarly, the plants and the microbes located at the plant's root, e.g., fungi and bacteria, communicate by means of chemical signals enabling mutual relationship for the plant and the microbes. That is, the plant releases metabolites to the soil, and the microbes provide nutrition and protection against pathogens and stress for the plant upon stimulation (Harkes et al., 2020). These interkingdom communication pathways are typically realized through the sharing of a common information carrier that is recognized by all involved species.

It is difficult to envision a scenario where a signal type of carriers is used to interconnect all entities, both natural and human-made, involved in a universal IoE. However, a universal transceiver supporting multi-modal communications, as shown in Figure 1, can overcome the interoperability challenge of the IoE by acting as a seamless interface between different types of networks.

Interfacing various networks of heterogeneous technologies via universal transceivers, that is the underlying theme of the IoE framework, is expected to open up unprecedented opportunities. For example, reprogramming biological communication systems for smart farming, can be realized by bridging different IoE technologies. More specifically, an Internet of Bio-Nano Things (IoBNT) (Akyildiz et al., 2015), which uses molecular communication modality to regulate the growth of certain microorganisms in soil that provide the plants with the immune response against the upcoming environmental changes, can be interconnected via an electromagnetic communication modality with an Internet of Drones (IoD) (Long et al., 2018; Cetinkaya and Merrett, 2020; Cetinkaya et al., 2020b) that provides real-time feedback by monitoring the crops having disease or stress. Similarly, an IoBNT using MC 


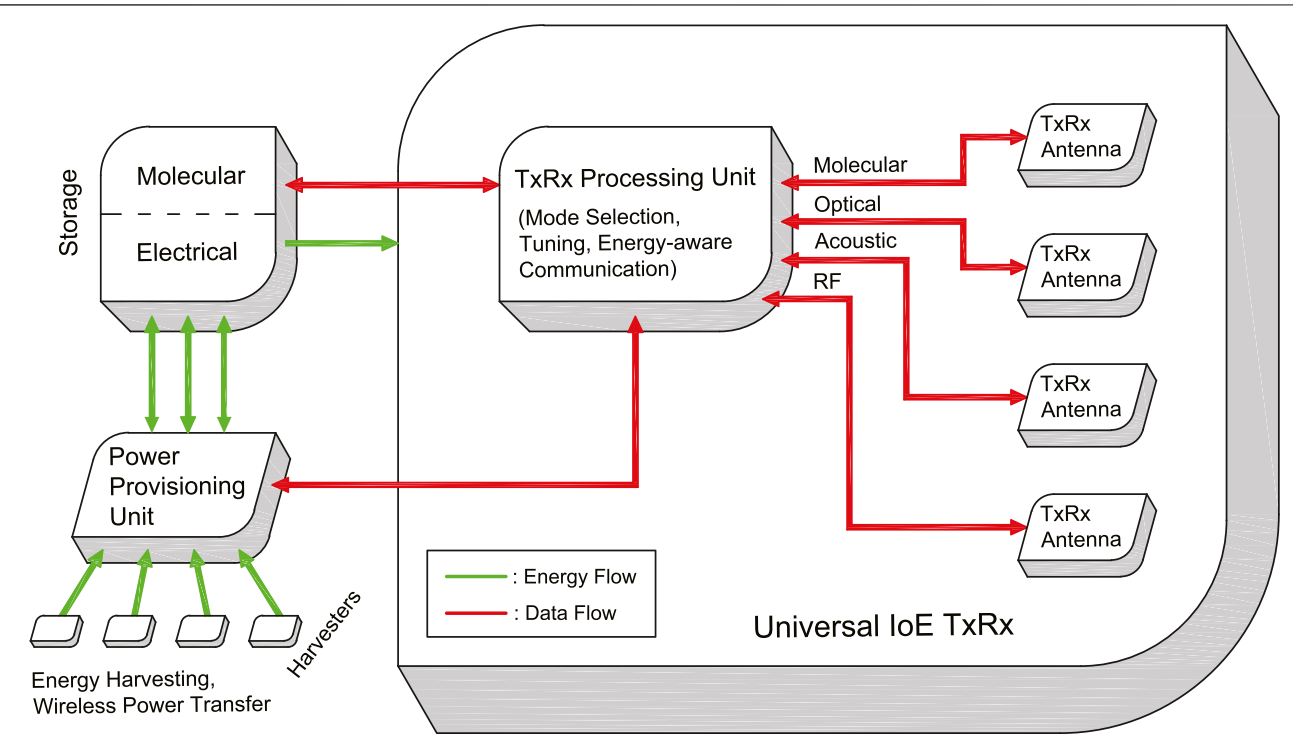

FIGURE 1 | An example architecture for a universal loE transceiver.

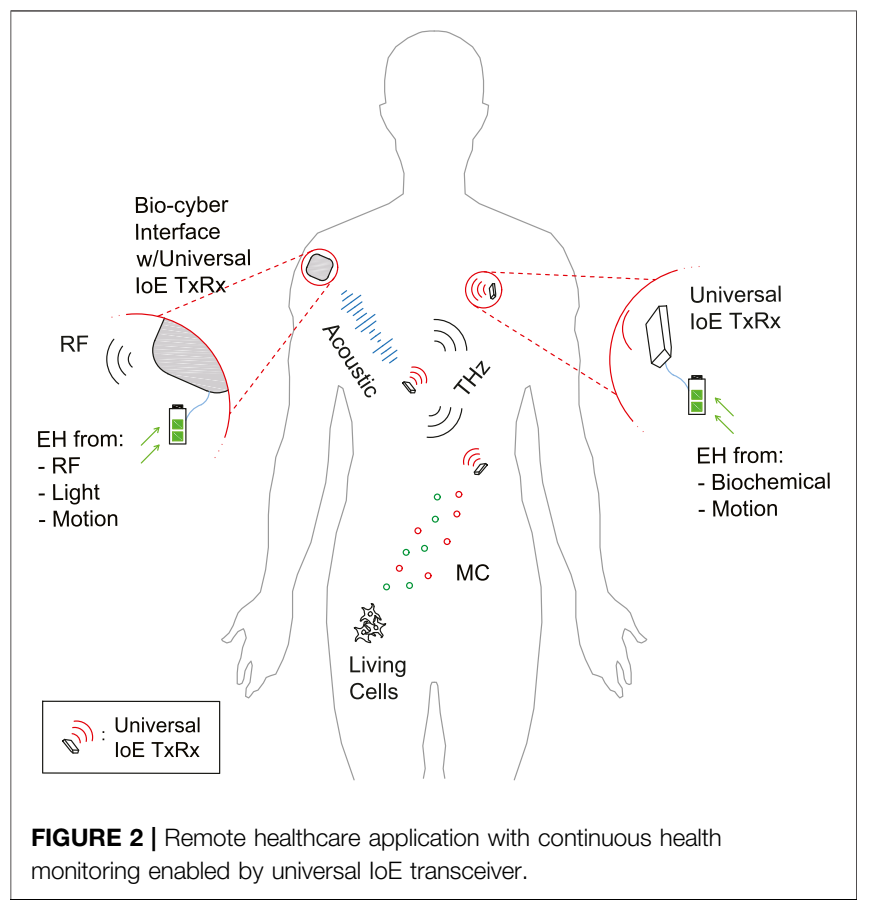

to communicate with the intra-body cells can be interconnected with a bio-cyber interface via multiple communication technologies suitable for the intra-body communication, e.g., $\mathrm{MC}, \mathrm{THz}$, and acoustic, for healthcare applications such as remote healthcare with continuous monitoring as illustrated in Figure 2. In particular, an IoBNT can monitor the concentrations of hormones or molecules in the blood, and transmit this information to the bio-cyber interface, which can connect to the conventional EM networks using RF modality so that the healthcare provider can remotely monitor the person's health condition (Khan et al., 2020a). Moreover, the neural interfaces and wearable sensor/actuator networks embedded with universal transceivers can be utilized in the transfer of senses, thoughts and skills non-verbally among individuals, i. e, Internet of People and Senses (IoPS) (Grau et al., 2014). In particular, a neural interface communicating with the neurons in the human brain can be interconnected to the other neural interfaces for the transfer of thoughts via low-latency and high-data-rate communication technologies, e.g., $\mathrm{THz}$ and optical, that can satisfy the requirements of such applications (Akan, 2021). The universal IoE transceivers can facilitate the cooperation of many other Internet of X's (IoXs), such as Internet of Vehicles (IoV) and Internet of Energy (IoEn), within the IoE framework for novel applications emerging from the close interaction between the heterogeneous technologies inherent in these IoXs.

\subsection{Ubiquitous Connectivity and Continuous Operation}

Varying environmental factors and dynamically changing application requirements demand flexibility and adaptation for maintaining reliable communication and continuous energy harvesting concurrently. The modular architecture and tunability, on which a universal IoE transceiver relies, can satisfy these requirements by enabling reconfigurability and adaptation. That is, a universal IoE transceiver either reconfigures its communication and/or energy harvesting modes to the one that is best suited for the environment and the application or opts for the most appropriate transmission/ reception and energy harvesting parameters. This can provide seamless coverage and uninterrupted operation everywhere.

There exist a number of communication technologies, each of which has various advantages and disadvantages for a particular application or an environment (see Table 1). The multi-modality 
TABLE 1 | Comparison of some of the key loE communication modalities.

\begin{tabular}{|c|c|c|c|}
\hline Communication modality & Medium & Data rate & Communication range \\
\hline Molecular & Intrabody, Airborne, Underwater & Very low & Low \\
\hline THz-band EM & Intrabody, Airborne & Very high & Low \\
\hline Acoustic & Intrabody, Airborne, Underwater & High & Moderate \\
\hline$R F$ & Airborne, Underwater & High & High \\
\hline Optical & Underwater, Intrabody & Very high & Moderate \\
\hline
\end{tabular}

support of an IoE device for communication can eliminate the shortcomings of a particular technology by allowing the utilization of advantage of another in a flexible manner. As an example, consider free space optical (FSO) and RF communications for outdoor applications. FSO communication can provide high data rates compared to RF communication. However, FSO communication is highly affected by the weather conditions such as fog and clouds, and atmospheric turbulence (Kaushal and Kaddoum, 2015). This can cause link failures or range reduction due to strong attenuation in hard weather conditions. To utilize the potentials of both technologies in the fullest extent and ensure reliability together, the IoE transceiver can employ both modalities adaptively and collaboratively using novel methods such as hybrid channel coding (Eslami et al., 2010).

Similarly, a universal transceiver can incorporate the advantages of multiple communication technologies, e.g., acoustic, optical and MC using buoyancy, for continuous operation in the underwater. Acoustic communication is a well known solution for underwater applications such as underwater habitat monitoring and submarine communication due to its robustness and relatively long-range support in the underwater. However, acoustic communication cannot support the applications requiring high-data rates such as high-quality video streaming due to the limited bandwidth. On the contrary, optical communication can provide extremely high data rates but suffers from the strong underwater attenuation limiting the communication range. Moreover, the optical links may not be always available because they require line-of-sight. For instance, in an underwater surveillance scenario based on the sensor network equipped with the universal IoE transceivers, videos or images can be streamed in real-time using optical links, and when the optical links are not available videos and images can be streamed using acoustic links nevertheless with low quality. Furthermore, both optical and acoustic communication may not work for the deep sea applications such as transmission of the location of black box on deep ocean floor, since optical and acoustic communication suffers from high attenuation and long channel multipath limiting the reliable communication in long distances, respectively. In such a scenario, the black box with a universal transceiver can use MC via buoyancy, which is a recently introduced delay-tolerant method for the deep sea applications (Guo et al., 2020), to transmit its location to the surface buoyant machine vertically. In MC via buoyancy, the injected information-carrying liquid, which is lighter than the ambient, is propelled by the buoyancy forces after losing the initial inertia force (Guo et al., 2020). Thus, when the buoyancy force is stronger than the horizontal ocean currents, buoyancybased MC links are suitable. On the other hand, MC via buoyancy still requires effective coding methods to improve the low transmission rates. In addition, according to the availability of the sensor network with the universal transceivers deployed nearby, the universal transceiver of the black box can opportunistically utilize acoustic or optical communication to communicate with the sensor nodes that can relay the information to the surface buoyant machine. Then, the surface buoyant machine relays the location of the black box to the drone network that can provide the rescue team with knowledge of the disaster area, as illustrated in Figure 3.

The dynamic tunability of transmission/reception parameters for a particular modality in the universal transceiver can help overcome challenges imposed by the environmental factors, affecting reliable operation. This can be exemplified by the case of MC, which uses molecules as the information carriers. The environmental factors, such as the chemical composition of the channel, dynamic flow conditions, and temperature, can affect the lifetime of the information carrying molecules and the bio-compatibility of the MC link (Kuscu et al., 2019). For example, considering an intrabody IoE application, a particular type of information carrying molecules might be safe only up to a certain level of concentration in blood, beyond which it may cause toxic effects for tissues and organs. A universal IoE transceiver can adapt to this condition by alternating the type of the carrier molecules within the set of molecule types harvested and stored in its molecular storage unit, as shown in Figure 1.

Continuous operation also requires exploitation of multiple energy sources due to the uncertainty inherent to these energy sources. A multi-modal energy harvesting unit sensing and exploiting diverse forms of energy resources available in the environment can alleviate this problem. The multi-modal energy harvesting capability will not only provide continuous operation but also increase the energy harvesting capacity of the universal IoE transceivers as discussed in Section 3.4.

\subsection{Increasing the Communication Performance}

Utilizing multi-modal communications for performance improvement is a emerging research topic (Famaey et al., 2018; Basu, 2021).

The current approaches are based on either using a particular modality at a time or using two different communication modalities simultaneously via multiplexing. However, with only these functionalities, the adaptation to the diverse 


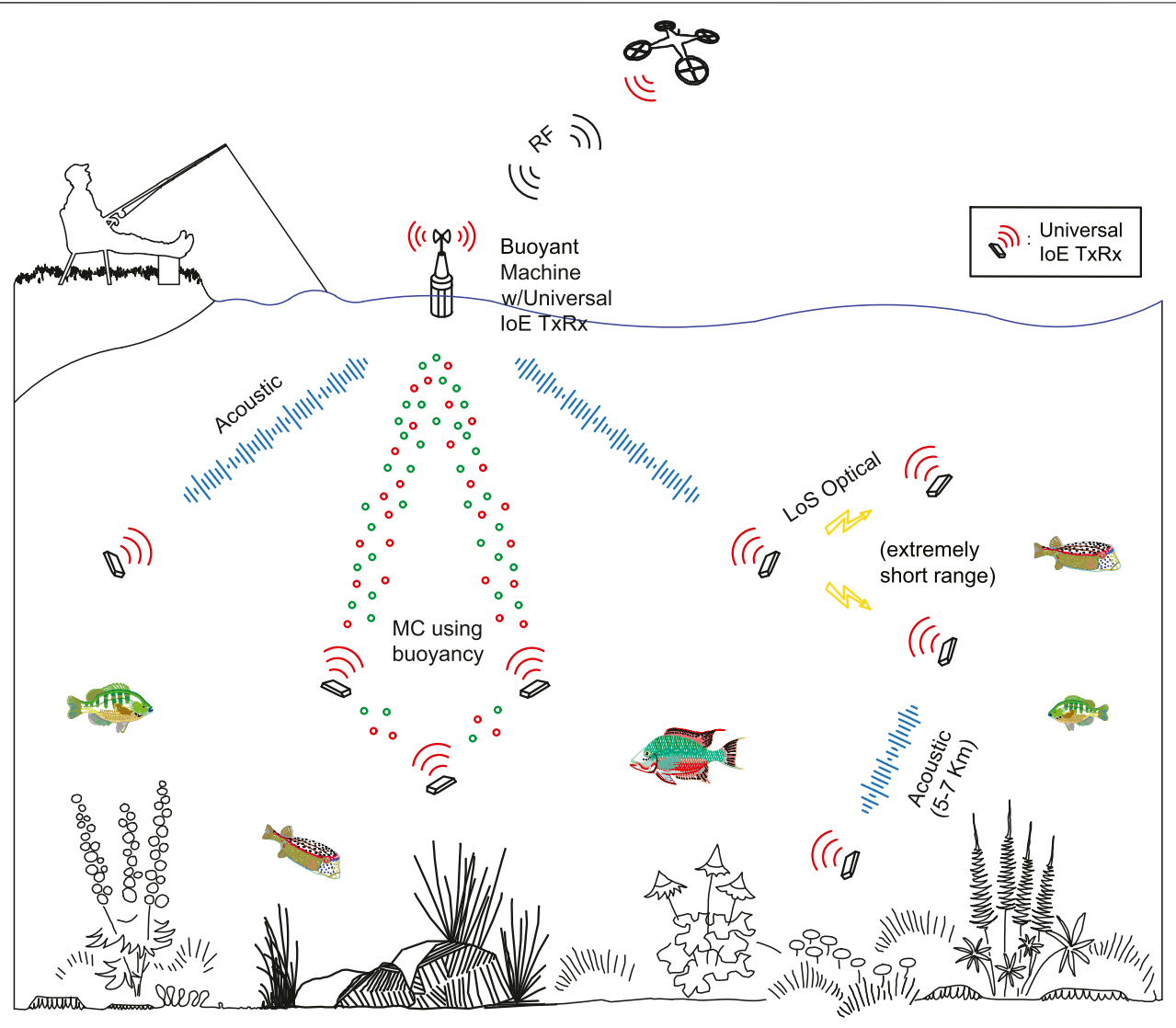

FIGURE 3 | Ubiquitous connectivity enabled by the universal loE transceivers for the underwater applications.

environments is not possible. A universal $\mathrm{IoE}$ architecture can simultaneously provide a combination of diverse functionalities including the aforementioned ones such as mode-switching, multi-modality and dynamic tuning of transmission/reception parameters, which can allow fullest adaptivity to the environment and/or application, and provide improved performance at the same time.

The functionalities of a universal IoE transceiver can pave the way for novel solutions tackling the performance impediments even in the arguably most challenging environments. For instance, there exist only a few technologies that can potentially support in-body communications, e.g., $\mathrm{MC}$ and Terahertz (THz)-band communications, due to the challenges imposed by the size constraints and physiological conditions. Considering an $\mathrm{MC}$ communication scenario involving multiple nanomachines sharing the same channel medium, multi-user interference (MUI) caused by the interfering molecules released by the other transmitters can degrade the performance significantly (Dinc and Akan, 2017). In case of a crowded MC network, the universal IoE transceiver can tune or reconfigure its communication mode opportunistically to minimize or avoid interference. For instance, the IoE transceiver can alternate the type of carrier molecules prior to transmission by dynamically sensing the channel in terms of concentration levels, similar to the cognitive radio methods in EM communications (Kuscu and
Akan, 2019). Alternatively, it can utilize an orthogonal communication modality, e.g., THz-band EM communications, to avoid MUI depending on the channel conditions. In addition, hybrid communication schemes multiplexing $\mathrm{MC}$ and THz-band communications can be devised to exploit orthogonality of these modalities collaboratively.

The functionalities of the universal IoE transceivers can also be leveraged in transmission control. For instance, in the case of MC, synchronization between the transmitter and the receiver is challenging because the feedback link from the receiver can cause huge overhead due to slow propagation of molecules (Lin et al., 2016). An IoE transceiver can employ different types of molecules for data transmission and synchronization (Jamali et al., 2017). Similarly, an IoE transceiver can use a communication modality in transmission control to assist another modality used in data transmission. A relevant example is the hybrid underwater optical/acoustic networks for real-time video streaming (Han, 2014). The optical data transmission is only possible when the optical modems are aligned properly. Therefore, until the alignment, while the optical links are not available, acoustic links can provide backup for transmission of data, and control signals, such as acknowledgements, for the optical modems. 


\subsection{Increasing the Energy Harvesting Capacity}

$\mathrm{EH}$ enables IoE transceivers to operate without requiring any external source of power. By assuring theoretically unlimited energy, $\mathrm{EH}$ aims to increase longevity and hence to replace batteries, in particular, which are not only capacity-limited but also maintenance-heavy and environmentally unfriendly. Despite its benefits, $\mathrm{EH}$ often fails to support periodic reporting due to the uncontrollable and unpredictable nature of the exploitable sources and the power-hungry communication capabilities of the IoE transceivers. Hence, the coexistence of different harvesting mechanisms is crucial to tackle the nondeterministic behavior of the $\mathrm{EH}$, achieving reliability in $\mathrm{IoE}$ communications.

Hybrid EH enables us to utilize ambient sources opportunistically and thus reduce the variance in the $\mathrm{EH}$ output thanks to the enhanced continuity in the energy input. If the deployment environment has multiple sources available together, the IoE transceiver can harvest them simultaneously or alternately. That often increases the amount of collected energy for a given time period, which expands the feasible energy tunnel enhancing the optimal energy allocation policy (Akan et al., 2018). If a large enough storage is employed, the optimal energy allocation policy of the hybrid $\mathrm{EH}$ IoE transceiver approximates to that of an unlimited battery, referring to perpetual and reliable operations at the highest transmission rate possible.

There are a plethora of studies combining different energy sources/harvesters in practice (Gupta, 2017). The prominent examples of this exploit either a single source (providing multiple energy streams, such as temperature gradients and vibrations generated on a pipe due to water flow) with different harvesting mechanisms, e.g., thermoelectric and piezoelectric generators, or multiple (diverse) sources, such as solar and wind power or acoustic waves and RF signals. The hybridization of sources should also increase the overall energy conversion efficiency of the system, compared to the sum of individual efficiencies, which can be achieved at the slight expanse of volume, cost, and complexity (Seol et al., 2016). Increasing the $\mathrm{EH}$ capacity is particularly important for applications in which the power provisioning process is intertwined with data communication. One example is the nano-devices harvesting molecules for energy generation, where some of those molecules are then used to transmit data. Hence, the IoE transceivers with multi-modal $\mathrm{EH}$ capabilities will increase the likelihood of energy generation from their surroundings by accommodating the necessary components, which offers numerous benefits especially for the multi-modal communications in the IoE domain.

\section{CHALLENGES AND POTENTIAL RESEARCH DIRECTIONS}

The fundamental properties of the universal IoE transceivers, i.e., multi-modality in communications and energy harvesting, modularity, tunability and scalability, bring many opportunities, some of which are discussed in the previous section; however, the realization of them poses unique challenges. In this section, some of these challenges along with the open research directions that can potentially address them are discussed. We first focus on the open problems in enabling the multi-modality in communications and energy harvesting. Our discussion is then extended to the adaptivity challenges in connection with the tunability property of the universal transceivers with regard to several aspects including channel sensing and energy awareness. We also provide an in-depth discussion on the miniaturization challenges to satisfy the scalability criterion of the universal transceivers.

\subsection{Multi-Modal Communications}

Integration of multiple communication modalities is a key feature of the universal IoE transceiver architectures, providing the IoE devices with the capability of seamless interfacing between heterogeneous networks and ubiquitous operation. On the other hand, multi-modality in communication poses two fundamental challenges, namely, reliable and information-efficient intermodality transduction, and cross-modality interference.

Inter-modality transduction is highly common in conventional communication networks. For instance, conversion between electrical and optical signals is the enabler of the global Internet through optical fiber links. Acoustic transceiver components such as acoustic signal generators and receivers also rely on transduction between acoustic and electrical signals. On the other hand, with the emergence of IoBNT, research efforts are increasingly focused on bridging conventional modalities, e.g., optical, magnetic and electrical, with the unconventional carriers of information such as molecules (Liu et al., 2017b; Grebenstein et al., 2019; VanArsdale et al., 2020). In this direction, proposed transduction methods are mainly based on synthetic biology products and tools, such as whole cell biosensors and optogenetic methods (Dixon et al., 2021). However, entirely biological nature of these transduction methods limits their application range to in vivo environments. On the other hand, the universal IoE transceiver consisting in the interface of conventional and unconventional communication modalities must be able to operate in various environments to support the universality of the IoE. To this end, investigation of novel nanomaterials which are compatible with a large range of environments and scales, and support transduction of different energy forms into each other could be a promising approach. Transduction performances of such materials in terms of information efficiency and delay also an issue requiring through investigation along with the hardware complexity burden that each modality adds. Moreover, multi-modality in communications comes with its unique challenges. For example, it is imperative to investigate cross-modality interference, which could significantly affect the communication performance, if two modalities are interlinked indirectly through their impact on communication channel or transceiver. In the following, we discuss some of these challenges and propose potential research directions. 


\subsubsection{Transduction}

Inter-modality transduction can be performed in two ways, namely transduction of all modalities to and from electrical domain, and direct transduction between different modalities without conversion to electrical domain. We envision that intermodality transduction in universal IoE transceiver architectures is based on the first one because current signal processing elements rely on electrical signals providing fast processing. Important challenges regarding the inter-modality transduction are transduction efficiency, processing delay, throughput mismatch between different modalities, and hardware complexity. Direct inter-modality transduction without conversion to electrical signal is also a potential approach that can be taken to address the aforementioned issues with the indirect transduction (Huang et al., 2016), (Hajizadegan et al., 2017).

Inter-modality transduction must be information efficient such that information loss during the transduction process should be minimized. In this direction, investigation of highperformance novel materials enabling transduction from a set of diverse modalities to electrical domain and from electrical domain to the modality of choice is crucial. Employing the same material for transduction of all modalities is also crucial for integrability. Moreover, for an efficient transduction, respective material parameters, which affect the material properties, must be optimized as well. Graphene and related materials (GRMs) are promising in this direction. Graphene is a two-dimensional carbon-based nanomaterial having unique electrical, optical, mechanical, thermal and chemical properties (Ferrari et al., 2015), which make it suitable for transduction of diverse modalities including acoustic, optical, RF/microwave, $\mathrm{THz}$ and molecular. The outstanding properties of graphene also enable sensitive and fast sensing of diverse modalities including light and molecules (Ferrari et al., 2015). Moreover, as a planar material graphene provides flexibility in a way that high integrability and compactness are possible (Ferrari et al., 2015).

\section{GRMs in Acoustic-Electrical Transduction}

Thanks to its high mechanical strength and ultra-thin structure, graphene is deemed promising as a wideband ultrasonic transducer as well as wide-frequency-response electrostatic acoustic transducer (Zhou et al., 2015; Zhou and Zettl, 2013). Thin structure of graphene-based diaphragm of acoustic transducers provides the broadening in the frequency spectrum of the generated or detected acoustic waves considering conventional diaphragms, and high mechanical strength of graphene makes graphene-based diaphragms robust to deformation (Zhou and Zettl, 2013). On the other hand, such acoustic transducers can have limitations in terms of the output quality. For instance, graphene-based acoustic wave generators provide limited output pressure density due to the loss caused by high sheet resistance of graphene (Xu et al., 2013). In this direction, thermo-acoustic graphene acoustic wave generators using thermal variations in the air near its surface instead of mechanical vibrations to generate acoustic waves offer a great potential because of their flatter output response at wide frequency range and the output power increasing linearly with the input power (Tu et al., 2019). In this direction, investigating the performance of graphene acoustic transducers in diverse environments, e.g., underwater, is also crucial for the IoE support.

\section{GRMs in EM-Electrical Transduction}

Graphene can also be used in wideband optical to electrical and electrical to optical conversion thanks to its electro-absorption and electro-refraction properties (Romagnoli et al., 2018). Due to its high conductivity, graphene can also be utilized in radiating and receiving RF and microwave signals. Although exfoliated and chemical vapor deposition (CVD)-based graphene sheets exhibit large surface resistance causing large losses at $\mathrm{RF} /$ microwave frequencies (Gomez-Diaz and Perruisseau-Carrier, 2012), it has been demonstrated that screen-printed graphene components can be utilized in RF sensing and transmission (Huang, 2016). Graphene also supports the tunable propagation of Surface Plasmon Polariton (SPP) waves at $\mathrm{THz}$ band (Liu et al., 2014), which are EM waves propagating along a metal-dielectric (or semiconductor-dielectric) interface formed through the interaction of EM field with the oscillation of surface charges. This capability of graphene has opened the way for the development of tunable graphene plasmonic modulators modulating SPP waves, and tunable graphene plasmonic nanoantennas converting SPP waves to EM waves and vice versa (Jornet and Akyildiz, 2014; Singh, 2016).

\section{GRMs in Molecular-Electrical Transduction}

Graphene can also be utilized in molecular-to-electrical and electrical-to-molecular transduction (Kuscu et al., 2019). Fieldeffect transistor-based biosensors (bioFETs) made of functionalized graphene have been shown to be promising in the transduction of biomolecular concentrations, e.g., present in blood, saliva and tissues (Szunerits and Boukherroub, 2018; Tsang, 2019), into electrical signals. There also exist porous graphene structures, which can be utilized as a membrane in drug reservoirs that can selectively release particular types of molecules through controllable pore sizes (Kuscu et al., 2019; Walker et al., 2017). In addition to GRMs, transition metal dichalcogenides (TMDs) are also emerging 2D layered materials offering great potential for inter-modality transduction (Manzeli, 2017). A particular member of TMDs is Molybdenum disulfide $\left(\mathrm{MoS}_{2}\right)$, which has attracted great attention because it is electrically and mechanically robust. In this direction, various applications of $\mathrm{MoS}_{2}$ have been reported including highly sensitive molecular-to-electrical conversion (Sarkar et al., 2014) and optical-to-electrical conversion (Lopez-Sanchez et al., 2013), and electro-optic modulators (Li et al., 2017; Sun et al., 2016). Black phosphorus (BP), which is the another class of $2 \mathrm{D}$ materials with the applications in optical detection and modulation, and molecule sensing (Zhou et al., 2017), can be potentially utilized in inter-modality transduction.

\section{Cross-Domain Matching}

The investigation of high-performance materials is also crucial to address transduction and processing delay issue. Graphene is also promising in this direction. Thanks to its exceptionally high 
carrier mobility (up to $\sim 10^{5} \mathrm{~cm}^{2} / \mathrm{V} \cdot \mathrm{s}$ at room-temperature (Purdie et al., 2018)), graphene can transform light as well as $\mathrm{THz}$ signals into electrical signal with ultrafast response (Mueller et al., 2010; Mittendorff et al., 2013). Graphene can also enable tunable ultrahigh-speed processing components for $\mathrm{THz}$-band communication such as frequency converters, mixers and modulators (Kovalev et al., 2021). Large surface specific area of graphene $\left(\sim 2,600 \mathrm{~m}^{2} / \mathrm{g}\right)$ provides rapid sensing of molecules as well (Yuan and Shi, 2013). It has been also demonstrated that graphene composites can be utilized in ultra-fast sensing of acoustic signals (Dinh Le, 2019).

Information throughput of different communication links might be different due to the diversity in employed communication methods, bringing the challenge of throughput-mismatch in interfacing heterogeneous networks. For instance, consider a IoBNT scenario where an intra-body nanonetwork employing MC interfaces with external networks via EM communication. Due to slow molecular diffusion and reaction dynamics, $\mathrm{MC}$ provides very low data rates compared to conventional EM communications. In such as scenario, when the external device sends high-rate data to an intra-body nanomachine with an universal IoE transceiver, the nanomachine may not relay the external input to the other intra-body nanomachines reliably because of the limited storage capacity, i. e, buffer overflow. Thus, novel transmission rate control techniques for limited storage capacity are required in this direction.

\section{Integration}

The integration of separate transduction, processing, and memory components for multiple communication modality can increase the hardware complexity of the universal IoE transceivers. In this regard, one of the potential research directions is the direct inter-modality transduction without conversion to electrical signals, which removes the necessity of processing in electrical domain, and also improves the transduction efficiency and delay. Alternatively, materials and methods enabling multi-functional capabilities, e.g., transduction, signal processing and memory, can be investigated towards reducing the complexity.

\subsubsection{Cross-Modality Interference}

As the universal IoE transceivers can use multiple communication modalities simultaneously, a novel performance limiting factor, namely the cross-modality interference emerges. For instance, when $\mathrm{MC}$ and $\mathrm{EM}$ communication are active at the same time, MC changes molecular composition of the medium, which in turn affects the propagation of EM waves in a way that molecules vibrate as a result of energy absorption from EM waves and causes the molecular absorption noise at the corresponding EM wave frequencies (Yang et al., 2020). The impact is drastic at $\mathrm{THz}$ and optical frequencies. Similarly, EM radiation can affect the performance of MC because the temperature fluctuations due to molecular absorption of EM energy affect molecular dynamics (Singh et al., 2020). Moreover, the molecular composition of the medium can change the reception/transmission performance of the transceiver components. For instance, absorption of interfering molecules on the graphene $\mathrm{THz}$ or optical antennas can change the refractive index of the antenna surface, which in turn affects the propagation of surface plasmonic waves (Rodrigo et al., 2015). A similar effect can be observed on graphene-oxidebased optical antennas, whose fluorescent properties are affected by the $\mathrm{pH}$ change in the medium due to the interfering chemical molecules (Gao, 2021). This challenge can be addressed by identifying all the possible cross-modality interferences and developing the necessary channel and network coding methods.

\subsection{Multimodal Energy Harvesting}

Leaving aside the continuous efforts to improve simplicity and hence energy efficiency of the communication methods, such as modulation and detection techniques (Kuscu et al., 2019), the most promising solution to enable self-sustaining operations is the integration of $\mathrm{EH}$ modalities into the IoE domain. $\mathrm{EH}$ has recently received tremendous research interest partly due to the energy needs set by the emerging IoE applications. Different application requirements and ever-changing medium conditions have proliferated the need for $\mathrm{EH}$ methodologies to achieve robust, continuous, and self-sustaining operations by reducing the impact of unpredictable and uncontrollable factors, i.e., the uncertainties. These are the options that have been considered for self-sustainable communication networks; thus, they are promising for integration into the IoE transceivers in a multimodal manner. Depending on the application environment and device architectures, various energy sources can be exploited by the IoE devices (Dinc et al., 2019; Jayakumar, 2014). For example, solar power, flow energy, vibrations, electromagnetic signals, and metabolic sources have been deemed feasible for harvesting (Akan et al., 2018). Despite being useful, using only one harvesting mechanism may not always be enough for the continuous operation of $\mathrm{IoE}$ devices. Hence, the research has looked towards hybridization of harvesters/resources.

\subsubsection{Hybrid Energy Harvesting Methods}

An interesting research direction in parallel with the wider IoE vision is towards hybrid $\mathrm{EH}$ systems exploiting multiple energy sources. We have previously investigated novel $\mathrm{EH}$ methods based on ambient electric field (Cetinkaya and Akan, 2017a; Cetinkaya and Akan, 2017b; Pehlivanoglu, 2018), and introduced energy-neutral Internet of Drones concept under the umbrella of IoE (Long et al., 2018). The aim here should be to combine several $\mathrm{EH}$ mechanisms for designing self-sustaining battery-less universal transceivers that can support various applications from micro-nano scale in-body applications to macro-scale airborne applications. Towards this objective, total energy consumption of universal transceiver should be estimated by considering energy requirements of different communication modalities and processing units. At the end, different $\mathrm{EH}$ and WPT techniques can be combined to provide the energy required for node operation, i.e., sensing and processing, as well as data communication between nodes and information gateways.

Concerning the intrabody and body area applications, human body stands as a vast source of energy in the form of mechanical vibrations resulting from body movements, respiration, 
heartbeat, and blood flow in vessels, thermal energy resulting from varying body temperature, and biochemical energy resulting from metabolic reactions and physiological processes (Dagdeviren et al., 2017). Literature now includes a multitude of successful applications of $\mathrm{EH}$ from human body to power miniature biomedical devices and implants. Some examples are thermoelectric EH from body heat for wearable devices (Leonov, 2013), piezoelectric EH from heartbeats (Amin Karami and Inman, 2012) and respiratory movements (Zheng et al., 2014) for pacemakers, as well as biochemical $\mathrm{EH}$ from human perspiration (Jia et al., 2013). These together with EH from chemical reactions within the body, such as glucose uptake, lactate release, and $\mathrm{pH}$ variations (Dagdeviren et al., 2017; Shi et al., 2018), can be exploited to power the IoE transceivers. Among the potential $\mathrm{EH}$ mechanisms, mechanical $\mathrm{EH}$ has attracted the most interest. Research in this field has gained momentum with the use of flexible piezoelectric nanomaterials, such as $\mathrm{ZnO}$ nanowires and lead zirconate titanate (PZT), in nanogenerators, enabling energy extraction from natural and artificial vibrations with frequencies ranging from very low frequencies $(<1 \mathrm{~Hz}$ ) up to several $\mathrm{kHz}$ (Wang and $\mathrm{Wu}, 2012$; Akyildiz and Jornet, 2010). A hybrid EH architecture is also proposed for IoE comprising modules for $\mathrm{EH}$ from light, mechanical, thermal, and EM sources (Akan et al., 2018).

\subsubsection{Wireless Power Transfer}

As explained previously, the universal IoE transceivers can sustain multi-modal $\mathrm{EH}$ capabilities by benefiting from ambient resources that are available in their deployment environment. In some cases, some of those sources can be dedicated, i.e., external sources delivering power to the IoE transceivers remotely, which refers to WPT. WPT has seen significant advances in recent years due to increasing need for replenishing the energy reservoir of IoT devices as well as wearable and implantable devices (Wagih, 2020). Various forms of WPT have been considered for powering medical implants (Agarwal et al., 2017; Ho et al., 2014). For example, near-field resonant inductive coupling (NRIC)-based WPT, the oldest WPT technique, has been widely-used for cochlear implants (Khan et al., 2020b; Manoufali, 2017). Other techniques include near-field capacitive coupling, mid- and far-field EM-based WPT, and acoustic WPT. The near-field capacitive and inductive coupling, however, is only efficient for distances on the order of transmitting and receiving device sizes, and for the right alignment of devices. Therefore, it might not be suitable for powering micro/nanoscale IoE tranceivers (Khan et al., 2020b). On the other hand, radiative mid- and farfield EM-based WPTs can have looser restrictions depending on the frequency of the EM waves.

Recent research on mmWave and $\mathrm{THz}$ rectennas suggests the use of high-frequency EM WPT techniques for power delivery (Rong et al., 2018). However, for intra-body applications, the higher absorption with increasing frequency and power restrictions should be taken into account. Nonetheless, simultaneous wireless information and power transfer techniques (SWIPT) utilizing THz-band have been investigated for EM nanonetworks (Feng et al., 2020; Rong et al., 2017). Similar SWIPT applications have been considered for MC, where the IoE devices use the received molecules for both decoding the information and harvesting energy (Guo et al., 2017; Deng et al., 2016). There are also applications of acoustic WPT for biomedical implants using external ultrasonic devices (Maleki et al., 2011; Larson and Towe, 2011). Although not practically implemented yet, ultrasonic $\mathrm{EH}$ has been also considered for powering IoE devices with piezoelectric transducers (Donohoe et al., 2015; Donohoe et al., 2017; Balasubramaniam et al., 2018).

It should be noted that both EH and WPT are still at their infancy especially for nano-molecular communications in the IoE domain. New research efforts focus on reducing the power requirements of IoE transceivers by using energy efficient protocols, conservation schemes and novel topologies, rather than focusing on EH and WPT mechanisms or their joint utilization (Cetinkaya et al., 2019). Therefore, it stands as a major open issue to utilize various $\mathrm{EH}$ and WPT mechanisms to create modular energy unit for the universal transceiver framework, such that different power extraction modalities can be configured depending on the application and medium of operation.

\subsection{Adaptivity and Tunability 4.3.1 Channel Sensing and Cognitive Radio}

Channel sensing is essential to enable adaptivity in universal transceiver architectures. The adaptive and opportunistic selection of the most proper communication modality and dynamic tuning of transmission and reception characteristics require dynamic channel sensing in multiple modalities and in a broadband manner. Broadband sensing refers to the sensing capability that can provide tunability property to the universal IoE transceivers in the maximum possible extent. For instance, in the case of MC, sensing as many different kinds of molecules as natural systems have is required for bio-compatibility. For EM communication, sensing at wide frequency range is needed for dynamic tunability. On the other, simultaneous channel sensing in different communication modalities may impose high complexity burden. These issues necessitate the development of low-complexity, reliable and broadband channel sensing techniques for each communication modality. Channel sensing techniques have been widely explored for RF (Yucek and Arslan, 2009; Ozger et al., 2018), optical wireless (Gong and Xu, 2015; Liu et al., 2017a; Arya and Chung, 2021) and acoustic communications (Baldo et al., 2008; Bicen et al., 2012), and similar investigations have just started for MC (Kuscu and Akan, 2019).

For the RF modality, spectrum sensing has received great attention because it is indispensable part of cognitive radio (CR) technology alleviating spectrum scarcity problem via dynamic spectrum access. CR capability can provide the universal transceivers with the adaptivity desired in a way that the universal transceivers can reconfigure their transmission parameters according to the available communication resources in the surroundings. Considering the heterogeneity of networks in the IoE environment, diverse communication technologies will utilize the RF spectrum. In this respect, one particular challenge is the identification of the signal types/ 
transceivers occupying the bands and their corresponding transmission parameters, e.g., carrier frequency, bandwidth, modulation type, transmission protocol, number of carriers (Eldemerdash et al., 2016), which is crucial to obtain spectrum awareness. Different from the classical signal identification techniques, deep-learning based identification does not require prior knowledge about the signals such as noise statistics, demanding extensive analysis (Zheng et al., 2020; Tekbiyık, 2020). Hence, deep learning-based identification techniques could be promising in this direction but the complexity burden should be further considered. Regarding spectrum sensing in RF spectrum, wideband and low-complexity compressed sensing techniques are available, thus can be utilized for the universal transceivers (Wei-Yu Chiu et al., 2012).

As the frequency usage is shifting towards mmWave and $\mathrm{THz}$ frequencies in EM communication, there is also need for ultrawideband spectrum sensing techniques. Considering the highsampling rate requirement of mmWave and $\mathrm{THz}$ band communication technologies, compressed sensing techniques exploiting the inherent sparsity of mmWave and $\mathrm{THz}$ signals can be utilized in universal transceivers (Brighente et al., 2020). Time-varying spectrum occupancy and high path losses affecting reliable sensing are the other issues relevant for channel sensing in $\mathrm{mmWave} / \mathrm{THz}$ communication modalities (Hamdaoui et al., 2020).

CR has also been considered for acoustic communication systems for efficient spectrum usage. The concerted effort in cognitive acoustic have been focused on underwater acoustic communications (Luo et al., 2014). The challenges identified in this direction include narrowband response of the acoustic transducers, long preamble of underwater acoustic modems, i.e., guard time or cyclic prefix signal inserted to overcome interference, and highly varying underwater channels (Luo et al., 2014). As discussed in Section 4.1, graphene-based acoustic transducers, which are suitable for wideband acoustic transduction (Zhou et al., 2015), offer a potential to address narrowband transduction issue. Moreover, further investigation considering channel conditions peculiar to other application environments different than the underwater channels is needed towards realizing cognitive acoustic for the universal transceivers.

Similar to the CR technology, the universal transceivers can use the MC channels opportunistically by sensing the type and concentration of molecules in the channel dynamically. For instance, if the channel is crowded with a specific type of molecule, the transceiver can use a different type of molecule to avoid MUI. However, sensing becomes challenging as the number of communication entities using MC, including bioentities using chemical signaling, in the medium increases because there might be many types of molecules in the medium. Thus, to maintain reliable communication and to minimize the possible harmful effects on surrounding bioprocesses, novel MC wideband sensing techniques recognizing as many as molecules are needed.

In addition to the modality-specific issues in channel sensing, there are novel issues such as sampling rate adjustment. Sampling rate can be different for different modalities. For instance, MC does not require high-rate sampling as the wideband RF spectrum sensing requires. However, in dynamic environments higher sampling rates may be needed. Hence, the dynamic optimization of the sampling rate is an open problem.

\subsubsection{Energy-Aware Communications}

The conventional way of ensuring the longevity for energyconstrained devices is to adopt energy-efficient communication techniques since the majority of the limited energy budget is consumed during data transmission. Radio optimization, clock synchronization, low-power sleep/wake-up, energy-efficient routing, and data reduction schemes are the main enablers of lengthened operation times (Cetinkaya et al., 2020b).

Energy-aware communications can be regarded as a subset of those techniques yet they have some differences in practice. For example, the low-power operation can be achieved through aggressive duty-cycling and/or wake-up radios by turning the radio on (for idle-listening or transmission) and off (for power saving) periodically (Longman et al., 2021). In similar, multi-path routing, data compression, and network coding schemes can be adopted during the system design stage to assure reliable communications on a budget. When the energy input is not constant, i.e., the device is creating its own energy via $\mathrm{EH}$, however, energy-aware communication techniques come into prominence. In such cases, the devices adapt to energy availability by changing their transmit power and/or sampling rate dynamically, besides updating their data queue by dropping some packets based on freshness or priority. The recently emerged intermittent or transient computing techniques, on the other hand, can assure application execution under scarce $\mathrm{EH}$ conditions through an incremental process at each power cycle based on the energy input (Sliper et al., 2020; Balsamo, 2020).

On the molecular level, energy-aware algorithms can be used for efficient coordination and movement $\mathrm{EH}$ nanonetworks, e.g., engineered bacteria tracking and eliminating harmful targets in the human body (Islam et al., 2020). Since the molecules harvested for energy generation can also transport information, the $\mathrm{EH}$ process directly governs data transmissions (Bafghi et al., 2018). Other approaches, such as feedback control, are also promising for energy-aware MC, especially in diffusion-based information transfer (Musa et al., 2020). By adopting these technologies, the IoE transceivers can achieve energy awareness in communication in concordance with the $\mathrm{EH}$ modality selection mechanism.

\subsubsection{EH Modality Selection}

As discussed earlier, adaptive hybridization of different energy forms is crucial for the IoE vision as it grants unique capabilities. However, the design of a hybrid EH system is not always straightforward (Weddell et al., 2013) since each harvester/ storage device often needs its own interface circuitry, which translates into increased cost and complexity.

Most of the communication modalities utilize electrical energy, which is stored in electrical storage shown in Figure 1. To ensure the flow, the system needs an input power conditioning/management/provisioning circuit to interface 
with the $\mathrm{EH}$ sources, alternate between harvesters and energy stores (molecular or electrical), or combine them when necessary. This circuit should include a rectifier for converting the collected energy into a utilizable form and preventing its flow back to the harvester(s), besides a DC-to-DC converter for maximum power tracking and providing the stable output required by the transceiver antennas. The MC modality of the IoE transceiver can also use the electrical energy for internal operations, such as sensing and processing. However, the IoE transceiver harvesting molecules need additional components, such as a chemical bank with basic building components to convert the harvested molecules into molecular information (Guo et al., 2017), which can be attached to the molecular storage.

Once established, the IoE transceiver can harvest energy opportunistically from any source available in its vicinity, swap between (or combine) harvesters or energy stores when the primary source disappears or when it changes its application environment, and probe its proximity using different sensors enabled/disabled according to the requirements of different tasks/ applications. In this way, universal, modular, and adaptive IoE systems can be devised.

As discussed, the EH modality selection can be either preplanned or adaptive to the ambient factors. One example is swapping sunlight to wind and/or rain drops when the weather in the deployment environment changes. The other case is the change of the environment itself, i.e., the system can harvest energy from heartbeat and/or blood flow instead of lactate or glucose when it moves within the body. These approaches often refer to online energy profiling, in which the IoE system adapts its operation to the current channel/medium state, thereby harvesting energy opportunistically. The conventional EH transceivers, however, commonly accommodate offline energy profiling, i.e., the medium-specific energy availability is known prior to operation. If the IoE transceiver employs the latter, it changes its application environment as it knows that a particular source will be available/in abundance in a particular location. This availability can be either due to perpetual bio-mechanical drivers, such as heartbeat, blood pressure, and breathing, or due to recurring events, e.g., periodically released hormones (Conn, 2013). Hence, the IoE system not only changes its medium but also its $\mathrm{EH}$ modality as a reflex to sustain reliable communications by getting the most out of the surrounding resources. That requires seamless coordination and efficient interaction of system components.

\subsection{Miniaturization}

The miniaturization trend at all levels of communication systems is being driven by the increasing number of connected devices. The miniaturization can provide key advantages including easy integrability, more functionality with lower footprint, increased performance, reduced power consumption and manufacturing cost. In the context of IoE, which aims at the ubiquitous connectivity of highly diverse entities, including bio/ nanomachines, the miniaturization of transceiver components to micro/nanoscales is indispensable. The future $6 \mathrm{G}$ networks will also be a part of the IoE environment, for which the frequency shift towards $\mathrm{THz}$ band in EM communication is inevitable (Dang et al., 2020). The usage of $\mathrm{THz}$ band necessitates downscaling the size of transceiver components to micro/ nanoscales. Moreover, nano and bio-nano networks using unconventional communications at nanoscales, such as MC, are important components of the $\mathrm{IoE}$, and interfacing these networks also requires miniaturization to micro/nanoscales. As such, universal transceivers must be integrable to a very large set of diverse devices and entities of varying scale and structures. Moreover, micro/nanoscale devices and components are known to be more energy-efficient in providing the similar functions as their macroscale counterparts. The miniaturization of the universal transceivers can also provide flexibility in their integration to various devices of different size scales to transform them into IoE devices. For example, micro/ nanoscale universal transceivers can be embedded into both micro/nanoscale biosensors and macroscale bio-cyber interfaces as part of an IoBNT application such as remote healthcare with continuous health monitoring based on IoBNT as illustrated in Figure 2.

In the past decades, silicon semiconductor technology has been the driving force for down-scaling the size of transistors so that they can be packed onto smaller and smaller chips. However, it is now long-evident that the silicon technology is reaching its physical limits. As silicon layers are getting thinner, electron flow through silicon channels is slowing down due to the surface imperfections that are scattering charges (Li, 2019). New novel nanomaterials with exceptional electrical, mechanical and biochemical properties, such as GRMs as discussed in Section 4.1, have emerged to overcome this limitation and to expand the diversity in functionalities in line with the More than Moore approach. However, there still exist substantial challenges with regard to growth and processing of these materials (Neumaier et al., 2019).

There also exist technical challenges to build some transceiver components with desired performances at micro/nanoscales. For instance, regarding conventional communications, e.g., RF and acoustic, shrinking the size of transceiver components such as antennas and modulators to micro/nanoscales while preserving their functions is still an issue because the operation in the micro/ nanoscale limits the carrier frequency to high-frequency ranges for these technologies. On the other hand, it should be noted that realizing universal IoE transceivers requires departure from the approaches aiming to optimize the performances of the transceiver components for individual modalities. Rather, the underlying theme of universal transceivers must be the maximization of the versatility stemming from multi-modality, scalability, and tunability, which can nevertheless be obtained by transceiver components suboptimally performing in individual modalities. Accordingly, in the following, we discuss the miniaturization challenges and potential research directions in developing miniaturized IoE universal transceiver components with regard to several modalities.

With the recent paradigm shift towards the usage of $\mathrm{THz}$ band, EM antennas are getting shrunk down to micro/nanoscales. Due to high losses introduced at $\mathrm{THz}$ frequencies by spreading, molecular absorption and scattering, the $\mathrm{THz}$ communication 

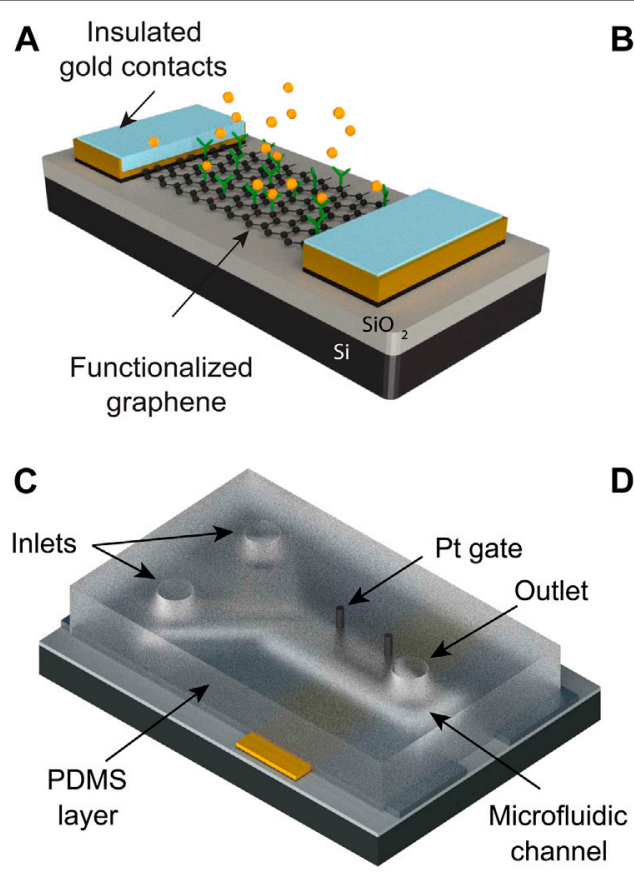

B

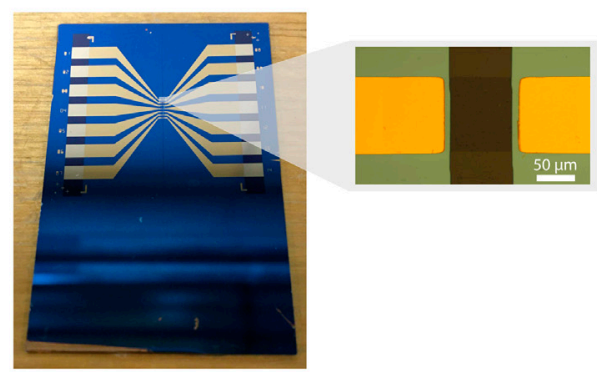

D

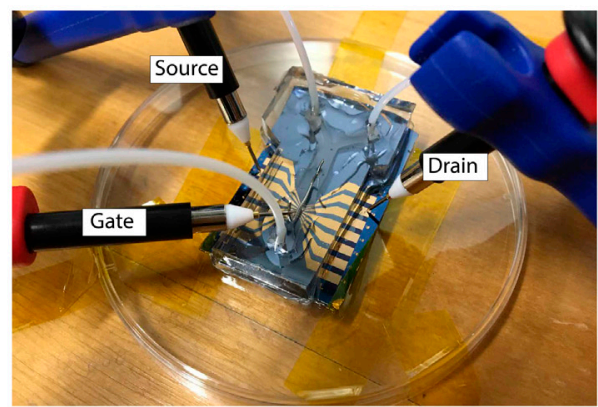

FIGURE 4 | Graphene-based micro/nanoscale MC receiver (Kuscu, 2021): (A) Conceptual drawing of MC receiver with functionalized graphene, (B) Fabricated $\mathrm{MC}$ receiver $\mathbf{( C , D )}$ Integration of the $\mathrm{MC}$ receiver into a microfluidic $\mathrm{MC}$ channel.

range is substantially limited. To overcome this limitation, multiinput-multi-output (MIMO) array architectures enabled by graphene plasmonic nano antennas have been proposed (Xu et al., 2014; Akyildiz and Jornet, 2016). Howeover, THz wave propagation introduces novel physical phenomena that can affect the performance of MIMO arrays. For instance, a THz beam may diverge from the direction of its modulation sidebands slightly due to frequency-dependent diffraction nature of $\mathrm{THz}$ signals (Ma et al., 2017). This may degrade the performance of a MIMO array in terms of bit error rate because different antennas may detect different spectral information (Ma et al., 2017). Thus, novel detection and demodulation methods are needed to overcome this limitation. Other important challenges towards $\mathrm{THz}$ communications with miniaturized transceivers are the efficient signal generation and detection. In this direction, concerted effort has been dedicated to solid-state electronics and photonics approaches (Sengupta et al., 2018). However, the electronics approach suffers from limited output power at $\mathrm{THz}$ frequencies and photonics approach based on quantum cascade lasers offers poor performance at room temperature (Nafari et al., 2018). In this direction, micro/nanoscale graphene transistors with extremely high carrier mobility can pave the way for the development of $\mathrm{THz}$-based signal generation and sensitive detection components (Bianco et al., 2015).

Silicon technology has achieved the miniaturization of optical communication components to microscale (Xu et al., 2005; Vahala, 2003). Recently, optical components utilizing SPPs have emerged for further miniaturization that are able to confine the light into nanometer scale. In this direction, plasmonic nanoantennas (Cohen et al., 2015), nanolasers ( $\mathrm{Lu}$ et al., 2012) and modulators (Klein et al., 2019) have been developed. The most pressing challenges in this direction are tuning plasmonic resonance frequency to higher frequencies, e.g., visible and near-infrared, which could be achieved through high level of doping (Yu et al., 2017), and integration of plasmonic components into non-plasmonic structures without high insertion losses (Haffner et al., 2015).

Regarding $\mathrm{MC}$, the first prototype of a nanoscale $\mathrm{MC}$ receiver, as illustrated in Figure 4, has been recently introduced in (Kuscu, 2021). This architecture incorporates the sensing mechanisms of electrical biosensors for detecting MC signals. Regarding the miniaturized MC transmitter component of the universal transceiver, as discussed in Section 4.1, porous graphene structures enabling controllable permeability across pores via electric field are promising for providing selective and tunable molecule transmission. On the other hand, high surface-to-volume ratio in nanopores exhibits new physical phenomenon that is not observed at larger scales. That is, surface charges become dominant in molecular transport by introducing electrostatic forces affecting the interactions between molecules and surfaces in liquids (Schoch et al., 2008). Thus, the effect of surface charges need to be considered in MC transmitter design. Molecule storage is also an important challenge regarding the miniaturization of MC transmitters. In this direction, stimuli-responsive hydrogels, which are also envisioned for smart drug delivery, are promising (Li and Mooney, 2016). Various type of molecules can be harvested from a fluidic medium by triggering the deswelling state in a hydrogel reservoir and loaded molecules can be transferred to $\mathrm{MC}$ transmitter antenna, i.e., tunable 
porous graphene membrane, by activating the swelling state through the application of a stimulus such as electrical field, magnetic field or light (Shi, 2019) in a controllable manner.

The miniaturization efforts regarding RF applications have focused on carbon-based materials, especially carbon nanotube (CNT) in the last two decades. CNTs have material properties including high mobility and small size that are suitable for miniaturized RF applications such as modulators, mixers, amplifiers (Schroter et al., 2013). Initial demonstrations of CNTs in RF applications were nanotube radios (Jensen et al., 2007; Rutherglen and Burke, 2007). Although the AM demodulator based on a single CNT in (Rutherglen and Burke, 2007) has small size, i.e., $1 \mathrm{e}-21 \mathrm{~m}^{3}$ of system volume, the overall volume of the system including the external antenna is of order $1 e-3 \mathrm{~m}^{3}$ (Burke and Rutherglen, 2010). In this direction, resonant CNT antennas can further reduce the overall system size because slow propagation of the waves on CNTs compared to free space provides a reduction in the resonant antenna size up to 100 times (Burke et al., 2006). Different from the radios in completely electrical nature, the operation of the nanotube radio implemented using a single CNT in (Jensen et al., 2007) is partly mechanical. Micro/nanometers long CNTs for nanoelectromechanical-based RF applications are suitable for the operation in $\mathrm{MHz}$ and $\mathrm{GHz}$ range, respectively. On the other hand, for both nanotube radios, the sensitivity is low such that receiving weak RF signals is not possible (Rutherglen et al., 2009). Thus, further research is needed in this direction towards realizing micro/nanoscale RF components for the universal IoE transceivers. Alternatively, magneto-electric (ME) materials are also promising to miniaturize RF components such as antennas to micro/nanoscales (Nan et al., 2017; Chen et al., 2020). For instance, instead of EM wave resonance, nanomechanical ME antennas operate at the acoustic resonance frequency. Since the acoustic wave length is smaller than the EM wave length at the same frequency, ME antennas can potentially provide dramatic miniaturization, i.e., hundreds to thousands of times smaller antennas, without performance degradation (Lin et al., 2018).

Two-dimensional nanomaterial technology have also opened the way for reducing the size of acoustic transceiver components, such as microphones, loudspeakers and earphones. In particular, graphene membranes that can generate and sense acoustic waves have received great interest due to their exceptional mechanical and electrical properties (Zhou et al., 2015; Zhou and Zettl, 2013; Tian, 2015). However, downscaling the dimensions of a graphene membrane, which is a vibrating element, to micro/nanoscales limits the operation frequency range to $\mathrm{MHz}-\mathrm{GHz}$ because the resonance frequency of a graphene membrane $f$ scales as $t / L^{2}$, where $t$ and $L$ is the thickness and the length of the graphene

\section{REFERENCES}

Agarwal, K., Jegadeesan, R., Guo, Y.-X., and Thakor, N. V. (2017). Wireless Power Transfer Strategies for Implantable Bioelectronics. IEEE Rev. Biomed. Eng. 10, 136-161. doi:10.1109/rbme.2017.2683520

Akan, O. B., Cetinkaya, O., Koca, C., and Ozger, M. (2018). Internet of Hybrid Energy Harvesting Things. IEEE Internet Things J. 5 (2), 736-746. doi:10.1109/ jiot.2017.2742663 membrane, respectively (Bunch et al., 2007). Thus, for the operation in the low frequency range, i.e., $\mathrm{Hz}-\mathrm{kHz}$, large membranes in millimeter scale are required (Al-Mashaal et al., 2018). Alternatively, based on the relation $t / L^{2}$, thick and small scale multilayer graphene membranes can be utilized in the low frequency range (Todorović, 2015). However, increased thickness may lower the sensitivity of the graphene membrane (Al-Mashaal et al., 2018). In addition, integrating graphene membrane to the universal transceivers can be challenging since it is a mechanical component. Therefore, further research is needed in this direction to realize micro/nanoscale broadband acoustic components for the universal transceivers.

\section{CONCLUSION}

In this paper, we introduced the concept of universal IoE transceiver that defines the transceiver architectures, which possess multi-modality in communication and energy harvesting, modularity, tunability, and scalability. Through several practical cases involving also non-conventional communication modalities, e.g., molecular, THz-band communications, which will be relevant in the upcoming IoE landscape, we argued that such universal transceivers that can be scaled down to micro/nanoscales could address the unique IoE challenges, such as interoperability, ubiquitous connectivity, energy efficiency, and miniaturization, and could open up further opportunities in diversifying the IoE applications. Along this direction, key physical layer challenges are outlined with potential solutions, which are centered around the use of novel nanomaterials, such as graphene, that manifest unique electrical, optical, mechanical and biochemical properties.

\section{AUTHOR CONTRIBUTIONS}

MC, OC, and MK developed the idea and wrote the manuscript, OBA supervised the research.

\section{FUNDING}

This work was supported in part by the AXA Research Fund (AXA Chair for Internet of Everything at Koç University), The Scientific and Technological Research Council of Turkey (TUBITAK) under Grant \#120E301 and Huawei Graduate Research Scholarship.

Akan, O. B. (2021). Information and Communication Theoretical Understanding and Treatment of Spinal Cord Injuries: State-Of-The-Art and Research Challenges. IEEE Rev. Biomed. Eng. doi:10.1109/rbme.2021.3056455

Akyildiz, I. F., and Jornet, J. M. (2010). Electromagnetic Wireless Nanosensor Networks. Nano Commun. Networks. 1 (1), 3-19. doi:10.1016/ j.nancom.2010.04.001

Akyildiz, I. F., and Jornet, J. M. (2016). Realizing Ultra-Massive MIMO $(1024 \times 1024)$ Communication in the $(0.06-10)$ Terahertz Band. Nano Commun. Networks. 8, 46-54. doi:10.1016/j.nancom.2016.02.001 
Akyildiz, I. F., Kak, A., and Nie, S. (2020). $6 \mathrm{~g}$ and Beyond: The Future of Wireless Communications Systems. IEEE Access. 8, 133995-134030. doi:10.1109/ access.2020.3010896

Akyildiz, I. F., Pierobon, M., and Balasubramaniam, S. (2019). Moving Forward With Molecular Communication: From Theory to Human Health Applications [Point of View]. Proc. IEEE. 107 (5), 858-865. doi:10.1109/jproc.2019.2913890

Akyildiz, I., Pierobon, M., Balasubramaniam, S., and Koucheryavy, Y. (2015). The Internet of Bio-Nano Things. IEEE Commun. Mag. 53 (3), 32-40. doi:10.1109/ mcom.2015.7060516

Al-Mashaal, A. K., Wood, G. S., Torin, A., Mastropaolo, E., Newton, M. J., Cheung, R., et al. (2018). Tunable Graphene-Polymer Resonators for Audio Frequency Sensing Applications. IEEE Sensors J. 19 (2), 465-473. doi:10.1109/ JSEN.2018.2877463

Amin Karami, M., and Inman, D. J. (2012). Powering Pacemakers From Heartbeat Vibrations Using Linear and Nonlinear Energy Harvesters. Appl. Phys. Lett. 100 (4), 042901. doi:10.1063/1.3679102

Arslan, H. (2007). Cognitive Radio, Software Defined Radio, and Adaptive Wireless Systems. Springer.

Arya, S., and Chung, Y. H. (2021). Spectrum Sensing for Optical Wireless Scattering Communications over Málaga Fading-A Cooperative Approach With Hard Decision Fusion. IEEE Trans. Commun. 69 (7), 4615-4631. doi:10.1109/TCOMM.2021.3073968

Bafghi, H. G., Gohari, A., Mirmohseni, M., Aminian, G., and Nasiri-Kenari, M. (2018). Diffusion-Based Molecular Communication With Limited Molecule Production Rate. IEEE Trans. Mol. Biol. Multi-Scale Commun. 4 (2), 61-72. doi:10.1109/tmbmc.2019.2895815

Balasubramaniam, S., Wirdatmadja, S. A., Barros, M. T., Koucheryavy, Y., Stachowiak, M., Jornet, J. M., et al. (2018). Wireless Communications for Optogenetics-Based Brain Stimulation: Present Technology and Future Challenges. IEEE Commun. Mag. 56 (7), 218-224. doi:10.1109/mcom.2018.1700917

Baldo, N., Casari, P., and Zorzi, M. (2008). "Cognitive Spectrum Access for Underwater Acoustic Communications," in ICC Workshops-2008 IEEE International Conference on Communications Workshops (IEEE), 518-523. doi:10.1109/iccw.2008.104

Balsamo, D. (2020). A Control Flow for Transiently Powered Energy Harvesting Sensor Systems. IEEE Sensors J. 20 (18), 10687-10695. doi:10.1109/ jsen.2020.2993213

Basagni, S., Valerio, V. D., Gjanci, P., and Petrioli, C. (2019). Marlin-q: MultiModal Communications for Reliable and Low-Latency Underwater Data Delivery. Ad Hoc Networks. 82, 134-145. doi:10.1016/j.adhoc.2018.08.003

Basu, S. S. (2021). "An Energy-Efficient Multi-Modal Iot System Leveraging Nb-Iot and Ble,", in 2020 IEEE International Conference on Internet of Things and Intelligence System (IoTaIS) (IEEE), 30-36.

Bianco, F., Perenzoni, D., Convertino, D., De Bonis, S. L., Spirito, D., Perenzoni, M., et al. (2015). Terahertz Detection by Epitaxial-Graphene Field-Effect-Transistors on Silicon Carbide. Appl. Phys. Lett. 107 (13), 131104. doi:10.1063/1.4932091

Bicen, A. O., Sahin, A. B., and Akan, O. B. (2012). Spectrum-Aware Underwater Networks: Cognitive Acoustic Communications. IEEE Veh. Technol. Mag. 7 (2), 34-40. doi:10.1109/mvt.2012.2190176

Brighente, A., Cerutti, M., Nicoli, M., Tomasin, S., and Spagnolini, U. (2020). Estimation of Wideband Dynamic Mmwave and Thz Channels for $5 \mathrm{~g}$ Systems and beyond. IEEE J. Select. Areas Commun. 38 (9), 2026-2040. doi:10.1109/ jsac.2020.3000889

Bunch, J. S., van der Zande, A. M., Verbridge, S. S., Frank, I. W., Tanenbaum, D. M., Parpia, J. M., et al. (2007). Electromechanical Resonators from Graphene Sheets. Science. 315 (5811), 490-493. doi:10.1126/science.1136836

Burke, P. J., Shengdong Li, S., and Zhen Yu, Z. (2006). Quantitative Theory of Nanowire and Nanotube Antenna Performance. IEEE Trans. Nanotechnology. 5 (4), 314-334. doi:10.1109/tnano.2006.877430

Burke, P., and Rutherglen, C. (2010). Towards a Single-Chip, Implantable Rfid System: Is a Single-Cell Radio Possible?. Biomed. Microdevices. 12 (4), 589-596. doi:10.1007/s10544-008-9266-4

Campagnaro, F. (2018). Optimal Transmission Scheduling in Small Multimodal Underwater Networks. IEEE Wireless Commun. Lett. 8 (2), 368-371. doi:10.1109/LWC.2018.2873329

Cetinkaya, O., and Akan, O. B. (2017a). Electric-Field Energy Harvesting From Lighting Elements for Battery-Less Internet of Things. IEEE Access. 5, 7423-7434. doi:10.1109/access.2017.2690968
Cetinkaya, O., and Akan, O. B. (2017b). Electric-Field Energy Harvesting in Wireless Networks. IEEE Wireless Commun. 24 (2), 34-41. doi:10.1109/ mwc.2017.1600215

Cetinkaya, O., Dinc, E., Koca, C., Merrett, G. V., and Akan, O. B. (2019). EnergyNeutral Wireless-Powered Networks. IEEE Wireless Commun. Lett. 8 (5), 1373-1376. doi:10.1109/lwc.2019.2918527

Cetinkaya, O., and Merrett, G. V. (2020). "Efficient Deployment of Uav-Powered Sensors for Optimal Coverage and Connectivity," in 2020 IEEE Wireless Communications and Networking Conference (WCNC) (IEEE), 1-6. doi:10.1109/wcnc45663.2020.9120738

Cetinkaya, O., Ozger, M., and Akan, O. B. (2020a). Internet of Energy Harvesting Cognitive Radios. Towards Cogn. IoT Networks. 125-150. doi:10.1007/978-3030-42573-9_9

Cetinkaya, O., Balsamo, D., and Merrett, G. V. (2020b). Internet of Mimo Things: Uav-Assisted Wireless-Powered Networks for Future Smart Cities. IEEE Internet Things M. 3 (1), 8-13. doi:10.1109/iotm.0001.1900064

Chen, H., Liang, X., Dong, C., He, Y., Sun, N., Zaeimbashi, M., et al. (2020). Ultracompact Mechanical Antennas. Appl. Phys. Lett. 117 (17), 170501. doi:10.1063/ 5.0025362

Cohen, M., Shavit, R., and Zalevsky, Z. (2015). Enabling High Efficiency Nanoplasmonics With Novel Nanoantenna Architectures. Scientific Rep., 5, 1-9. doi:10.1038/srep17562

Conn, P. M. (2013). Pulsatility in Neuroendocrine Systems. Academic Press.

Dagdeviren, C., Li, Z., and Wang, Z. L. (2017). Energy Harvesting From the Animal/Human Body for Self-Powered Electronics. Annu. Rev. Biomed. Eng. 19, 85-108. doi:10.1146/annurev-bioeng-071516-044517

Dang, S., Amin, O., Shihada, B., and Alouini, M.-S. (2020). What Should 6g Be? Nat. Electron. 3 (1), 20-29. doi:10.1038/s41928-019-0355-6

Deng, Y., Guo, W., Noel, A., Nallanathan, A., and Elkashlan, M. (2016). Enabling Energy Efficient Molecular Communication via Molecule Energy Transfer. IEEE Commun. Lett. 21 (2), 254-257. doi:10.1109/ LCOMM.2016.2624727

Dinc, E., and Akan, O. B. (2017). Theoretical Limits on Multiuser Molecular Communication in Internet of Nano-Bio Things. IEEE Trans.on Nanobioscience. 16 (4), 266-270. doi:10.1109/tnb.2017.2692646

Dinc, E., Kuscu, M., Bilgin, B. A., and Akan, O. B. (2019). Internet of Everything. IGI Glob., 1-30. doi:10.4018/978-1-5225-7332-6.ch001

Dinh Le, T.-S. (2019). Ultrasensitive Anti-interference Voice Recognition by BioInspired Skin-Attachable Self-Cleaning Acoustic Sensors. ACS nano. 13 (11), 13 293-313 303. doi:10.1021/acsnano.9b06354

Dixon, T. A., Williams, T. C., and Pretorius, I. S. (2021). Bioinformational Trends in Grape and Wine Biotechnology. Trends Biotechnol. 12 (1), 1-12. doi:10.1016/ j.tibtech.2021.05.001

Donohoe, M., Balasubramaniam, S., Jennings, B., and Miquel Jornet, J. (2015). Powering In-Body Nanosensors With Ultrasounds. IEEE Trans. Nanotechnology. 15 (2), 151-154. doi:10.1109/TNANO.2015.2509029

Donohoe, M., Jennings, B., Jornet, J. M., and Balasubramaniam, S. (2017). Nanodevice Arrays for Peripheral Nerve Fascicle Activation Using Ultrasound Energy-Harvesting. IEEE Trans. Nanotechnology. 16 (6), 919-930. doi:10.1109/tnano.2017.2723658

Eldemerdash, Y. A., Dobre, O. A., and Öner, M. (2016). Signal Identification for Multiple-Antenna Wireless Systems: Achievements and Challenges. IEEE Commun. Surv. Tutorials. 18 (3), 1524-1551. doi:10.1109/comst.2016.2519148

Eslami, A., Vangala, S., and Pishro-Nik, H. (2010). Hybrid Channel Codes for Efficient Fso/rf Communication Systems. IEEE Trans. Commun. 58 (10), 2926-2938. doi:10.1109/tcomm.2010.082710.090195

Famaey, J., Berkvens, R., Ergeerts, G., Poorter, E. D., Abeele, F. V. d., Bolckmans, T., et al. (2018). Flexible Multimodal Sub-gigahertz Communication for Heterogeneous Internet of Things Applications. IEEE Commun. Mag. 56 (7), 146-153. doi:10.1109/mcom.2018.1700655

Feng, L., Ali, A., Iqbal, M., Ali, F., Raza, I., Hameed Saddiqui, M., et al. (2020). Dynamic Wireless Information and Power Transfer Scheme for NanoEmpowered Vehicular Networks. IEEE Trans. Intell. Transportation Syst., 1-12. doi:10.1109/TITS.2020.3020254

Ferrari, A. C., Bonaccorso, F., Fal'ko, V., Novoselov, K. S., Roche, S., Bøggild, P., et al. (2015). Science and Technology Roadmap for Graphene, Related TwoDimensional Crystals, and Hybrid Systems. Nanoscale. 7 (11), 4598-4810. doi:10.1039/c4nr01600a 
Foster, J. A., and McVey Neufeld, K.-A. (2013). Gut-Brain Axis: How the Microbiome Influences Anxiety and Depression. Trends Neurosciences. 36 (5), 305-312. doi:10.1016/j.tins.2013.01.005

Gao, X. (2021). Graphene and its Derivatives-Based Optical Sensors. Front. Chem. 9 (5), 615164. doi:10.3389/fchem.2021.615164

Gomez-Diaz, J. S., and Perruisseau-Carrier, J. (2012). "Microwave to Thz Properties of Graphene and Potential Antenna Applications," in 2012 International Symposium on Antennas and Propagation (ISAP) (IEEE), 239-242.

Gong, C., and Xu, Z. (2015). Temporal Spectrum Sensing for Optical Wireless Scattering Communications. J. Lightwave Technol. 33 (18), 3890-3900. doi:10.1109/jlt.2015.2442232

Grau, C., Ginhoux, R., Riera, A., Nguyen, T. L., Chauvat, H., Berg, M., et al. (2014). Conscious Brain-To-Brain Communication in Humans Using Non-Invasive Technologies. PloS one. 9 (8), e105225. doi:10.1371/journal.pone.0105225

Grebenstein, L., Kirchner, J., Peixoto, R. S., Zimmermann, W., Irnstorfer, F., Wicke, W., et al. (2019). Biological Optical-To-Chemical Signal Conversion Interface: A Small-Scale Modulator for Molecular Communications. IEEE Trans. Nanobioscience. 18 (1), 31-42. doi:10.1109/TNB.2018.2870910

Guo, W., Atthanayake, I., and Thomas, P. (2020). "Vertical Underwater Molecular Communications via Buoyancy: Gaussian Velocity Distribution of Signal," in ICC 2020-2020 IEEE International Conference on Communications (ICC) (IEEE), 1-6. doi:10.1109/icc40277.2020.9148765

Guo, W., Deng, Y., Birkan Yilmaz, H., Farsad, N., Elkashlan, M., and Eckford, A. (2017). Smiet: Simultaneous Molecular Information and Energy Transfer. IEEE Wireless Commun. 25 (1), 106-113. doi:10.1109/MWC.2017.1600308

Guoqiang, S. (2013). “Design and Implementation of a Smart Iot Gateway,” in 2013 IEEE International Conference on Green Computing and Communications and IEEE Internet of Things and IEEE Cyber, Physical and Social Computing (IEEE), 720-723. doi:10.1109/greencom-ithings-cpscom.2013.130

Gupta, R. K. (2017). Broadband Energy Harvester Using Non-Linear Polymer spring and Electromagnetic/Triboelectric Hybrid Mechanism. Scientific Rep. 7 (1), 1-13. doi:10.1038/srep41396

Haffner, C., Heni, W., Fedoryshyn, Y., Niegemann, J., Melikyan, A., Elder, D. L., et al. (2015). All-Plasmonic Mach-Zehnder Modulator Enabling Optical HighSpeed Communication at the Microscale. Nat. Photon. 9 (8), 525-528. doi:10.1038/nphoton.2015.127

Hajizadegan, M., Sakhdari, M., Zhu, L., Cui, Q., Huang, H., Cheng, M. M. C., et al. (2017). Graphene Sensing Modulator: Toward Low-Noise, Self-Powered Wireless Microsensors. IEEE Sensors J. 17 (22), 7239-7247. doi:10.1109/ jsen.2017.2737699

Hamdaoui, B., Khalfi, B., and Zorba, N. (2020). Dynamic Spectrum Sharing in the Age of Millimeter-Wave Spectrum Access. IEEE Netw. 34 (5), 164-170. doi:10.1109/mnet.011.1900581

Han, S. (2014). "Real-Time Video Streaming From mobile Underwater Sensors," in Proceedings of the International Conference on Underwater Networks \& Systems,, 1-8. doi:10.1145/2671490.2674582

Harkes, P., van Steenbrugge, J. J. M., van den Elsen, S. J. J., Suleiman, A. K. A., de Haan, J. J., Holterman, M. H. M., et al. (2020). Shifts in the Active Rhizobiome Paralleling Low meloidogyne Chitwoodi Densities in Fields Under Prolonged Organic Soil Management. Front. Plant Sci. 10, 1697. doi:10.3389/ fpls.2019.01697

Ho, J. S., Yeh, A. J., Neofytou, E., Kim, S., Tanabe, Y., Patlolla, B., et al. (2014). Wireless Power Transfer to Deep-Tissue Microimplants. Proc. Natl. Acad. Sci. 111 (22), 7974-7979. doi:10.1073/pnas.1403002111

Huang, H., Tao, L., Liu, F., Ji, L., Hu, Y., Cheng, M. M., et al. (2016). ChemicalSensitive Graphene Modulator With a Memory Effect for Internet-Of-Things Applications. Microsyst Nanoeng. 2 (1), 16018-16019. doi:10.1038/ micronano. 2016.18

Huang, X. (2016). Graphene Radio Frequency and Microwave Passive Components for Low Cost Wearable Electronics. 2D Mater. 3 (2), 025021. doi:10.1088/2053-1583/3/2/025021

Islam, N., Pal, S., Balasubramaniam, S., and Misra, S. (2020). Energy-Aware Tracking of Mobile Targets by Bacterial Nanonetworks. IEEE Trans. Mobile Comput. 20, 2808. doi:10.1109/TMC.2020.2990134

Jamali, V., Ahmadzadeh, A., and Schober, R. (2017). “Symbol Synchronization for Diffusive Molecular Communication Systems,," in 2017 IEEE International
Conference on Communications (ICC) (IEEE), 1-7. doi:10.1109/ icc.2017.7996907

Jayakumar, H. (2014). "Powering the Internet of Things," in Proceedings of the 2014 international symposium on Low power electronics and design, 375-380. doi: $10.1145 / 2627369.2631644$

Jensen, K., Weldon, J., Garcia, H., and Zettl, A. (2007). Nanotube Radio. Nano Lett. 7 (11), 3508-3511. doi:10.1021/nl0721113

Jia, W., Valdés-Ramírez, G., Bandodkar, A. J., Windmiller, J. R., and Wang, J. (2013). Epidermal Biofuel Cells: Energy Harvesting From Human Perspiration. Angew. Chem. Int. Ed. 52 (28), 7233-7236. doi:10.1002/anie.201302922

Jornet, J. M., and Akyildiz, I. F. (2014). "Graphene-Based Plasmonic NanoTransceiver for Terahertz Band Communication," in The 8th European conference on antennas and propagation (EuCAP 2014) (IEEE), 492-496. doi:10.1109/eucap.2014.6901799

Kamalinejad, P., Mahapatra, C., Sheng, Z., Mirabbasi, S., M. Leung, V. C., and Guan, Y. L. (2015). Wireless Energy Harvesting for the Internet of Things. IEEE Commun. Mag. 53 (6), 102-108. doi:10.1109/mcom.2015.7120024

Khan, T., Civas, M., Cetinkaya, O., Abbasi, N. A., and Akan, O. B. (2020). Nanosensor Networks for Smart Health Care. Nanosensors for Smart Cities., 387-403. doi:10.1016/b978-0-12-819870-4.00022-0

Khan, S. R., Pavuluri, S. K., Cummins, G., and Desmulliez, M. P. Y. (2020b). Wireless Power Transfer Techniques for Implantable Medical Devices: A Review. Sensors. 20 (12), 3487. doi:10.3390/s20123487

Klein, M., Badada, B. H., Binder, R., Alfrey, A., McKie, M., Koehler, M. R., et al. (2019). 2d Semiconductor Nonlinear Plasmonic Modulators. Nat. Commun. 10 (1), 3264-3267. doi:10.1038/s41467-019-11186-w

Kaushal, H., and Kaddoum, G. (2015). Free Space Optical Communication: Challenges and Mitigation Techniques, arXiv preprint arXiv:1506.04836

Kovalev, S., Hafez, H. A., Tielrooij, K.-J., Deinert, J.-C., Ilyakov, I., Awari, N., et al. (2021). Electrical Tunability of Terahertz Nonlinearity in Graphene. Sci. Adv. 7 (15), eabf9809. doi:10.1126/sciadv.abf9809

Koucheryavy, Y. (2021). A Review on Bio-Cyber Interfaces for Intrabody Molecular Communications Systems. arXiv preprint arXiv:2104.14944

Kuscu, M., and Akan, O. B. (2019). Channel Sensing in Molecular Communications With Single Type of Ligand Receptors. IEEE Trans. Commun. 67 (10), 6868-6884. doi:10.1109/tcomm.2019.2933202

Kuscu, M., and Akan, O. B. (2015). "Modeling and Analysis of Sinw Biofet as Molecular Antenna for Bio-Cyber Interfaces towards the Internet of BioNanothings,"in 2015 IEEE 2nd World Forum on Internet of Things (WFIoT) (IEEE), 669-674. doi:10.1109/wf-iot.2015.7389134

Kuscu, M., Dinc, E., Bilgin, B. A., Ramezani, H., and Akan, O. B. (2019). Transmitter and Receiver Architectures for Molecular Communications: A Survey on Physical Design With Modulation, Coding, and Detection Techniques. Proc. IEEE. 107 (7), 1302-1341. doi:10.1109/jproc.2019.2916081

Kuscu, M. (2021). Fabrication and Microfluidic Analysis of Graphene-Based Molecular Communication Receiver for Internet of Nano Things (Iont). Scientific Reports.

Larson, P. J., and Towe, B. C. (2011). "Miniature Ultrasonically Powered Wireless Nerve Cuff Stimulator," in 2011 5th International IEEE/EMBS Conference on Neural Engineering (IEEE), 265-268.

Leonov, V. (2013). Thermoelectric Energy Harvesting of Human Body Heat for Wearable Sensors. IEEE Sensors J. 13 (6), 2284-2291. doi:10.1109/ jsen.2013.2252526

Li, B., Zu, S., Zhou, J., Jiang, Q., Du, B., Shan, H., et al. (2017). Single-Nanoparticle Plasmonic Electro-Optic Modulator Based on Mos2 Monolayers. ACS nano. 11 (10), 9720-9727. doi:10.1021/acsnano.7b05479

Li, J., and Mooney, D. J. (2016). Designing Hydrogels for Controlled Drug Delivery. Nat. Rev. Mater. 1 (12), 1-17. doi:10.1038/natrevmats.2016.71

Lin, H., Zaeimbashi, M., Sun, N., Liang, X., Chen, H., and Dong, C. (2018). "Nems Magnetoelectric Antennas for Biomedical Application," in 2018 IEEE International Microwave Biomedical Conference (IMBioC) (IEEE), 13-15. doi:10.1109/imbioc.2018.8428867

Lin, L., Yang, C., Ma, M., Ma, S., and Yan, H. (2016). A Clock Synchronization Method for Molecular Nanomachines in Bionanosensor Networks. IEEE Sensors J. 16 (19), 7194-7203. doi:10.1109/jsen.2016.2591823

Liu, S., Zhang, C., Hu, M., Chen, X., Zhang, P., Gong, S., et al. (2014). Coherent and Tunable Terahertz Radiation From Graphene Surface Plasmon Polaritons 
Excited by an Electron Beam. Appl. Phys. Lett. 104 (20), 201104. doi:10.1063/ 1.4879017

Liu, X., Gong, C., and Xu, Z. (2017a). Sequential Detection for Optical Wireless Scattering Communication. J. Opt. Commun. Netw. 9 (9), D86-D95. doi:10.1364/jocn.9.000d86

Liu, Y., Tsao, C. Y., Kim, E., Tschirhart, T., Terrell, J. L., Bentley, W. E., et al. (2017b). Using a Redox Modality to Connect Synthetic Biology to Electronics: Hydrogel-Based Chemo-Electro Signal Transduction for Molecular Communication. Adv. Healthc. Mater. 6 (1), 1600908. doi:10.1002/ adhm.201600908

Long, T., Ozger, M., Cetinkaya, O., and Akan, O. B. (2018). Energy Neutral Internet of Drones. IEEE Commun. Mag. 56 (1), 22-28. doi:10.1109/ mcom.2017.1700454

Longman, E., Cetinkaya, O., El-Hajjar, M., and Merrett, G. V. (2021). "Wake-up Radio-Enabled Intermittently-Powered Devices for Mesh Networking: A Power Analysis," in 2021 IEEE 18th Annual Consumer Communications \& Networking Conference (CCNC) (IEEE), 1-6. doi:10.1109/ ccnc49032.2021.9369557

Lopez-Sanchez, O., Lembke, D., Kayci, M., Radenovic, A., and Kis, A. (2013). Ultrasensitive Photodetectors Based on Monolayer MoS2. Nat. Nanotech. 8 (7), 497-501. doi:10.1038/nnano.2013.100

Lu, Y.-J., Kim, J., Chen, H.-Y., Wu, C., Dabidian, N., Sanders, C. E., et al. (2012). Plasmonic Nanolaser Using Epitaxially Grown Silver Film. Science. 337 (6093), 450-453. doi:10.1126/science.1223504

Luo, Y., Pu, L., Zuba, M., Peng, Z., and Cui, J.-H. (2014). Challenges and Opportunities of Underwater Cognitive Acoustic Networks. IEEE Trans. Emerg. Top. Comput. 2 (2), 198-211. doi:10.1109/tetc.2014.2310457

Li, M.-Y. (2019). How 2d Semiconductors Could Extend Moore's Law

Ma, J., Karl, N. J., Bretin, S., Ducournau, G., and Mittleman, D. M. (2017). Frequency-Division Multiplexer and Demultiplexer for Terahertz Wireless Links. Nat. Commun. 8 (1), 729-738. doi:10.1038/s41467017-00877-x

Maleki, T., Cao, N., Hyun Song, S., Kao, C., Song-Chu, K., and Ziaie, B. (2011). An Ultrasonically Powered Implantable Micro-Oxygen Generator (Imog). IEEE Trans. Biomed. Eng. 58 (11), 3104-3111. doi:10.1109/tbme.2011.2163634

Manoufali, M. (2017). Near-Field Inductive-Coupling Link to Power a ThreeDimensional Millimeter-Size Antenna for Brain Implantable Medical Devices. IEEE Trans. Biomed. Eng. 65 (1), 4-14. doi:10.1109/TBME.2017.2778729

Manzeli, S. (2017). 2d Transition Metal Dichalcogenides. Nat. Rev. Mater. 2 (8), 1-15. doi:10.1038/natrevmats.2017.33

Mittendorff, M., Winnerl, S., Kamann, J., Eroms, J., Weiss, D., Schneider, H., et al. (2013). Ultrafast Graphene-Based Broadband Thz Detector. Appl. Phys. Lett. 103 (2), 021113. doi:10.1063/1.4813621

Mueller, T., Xia, F., and Avouris, P. (2010). Graphene Photodetectors for HighSpeed Optical Communications. Nat. Photon. 4 (5), 297-301. doi:10.1038/ nphoton. 2010.40

Musa, V., Piro, G., Grieco, L. A., and Boggia, G. (2020). A Lean Control Theoretic Approach to Energy-Harvesting in Diffusion-Based Molecular Communications. IEEE Commun. Lett. 24 (5), 981-985. doi:10.1109/ lcomm.2020.2972900

Nafari, M., Aizin, G. R., and Jornet, J. M. (2018). Plasmonic Hemt Terahertz Transmitter Based on the Dyakonov-Shur Instability: Performance Analysis and Impact of Nonideal Boundaries. Phys. Rev. Appl. 10 (6), 064025. doi:10.1103/physrevapplied.10.064025

Nakano, T., Kobayashi, S., Suda, T., Okaie, Y., Hiraoka, Y., and Haraguchi, T. (2014). Externally Controllable Molecular Communication. IEEE J. Select. Areas Commun. 32 (12), 2417-2431. doi:10.1109/jsac.2014.2367667

Nan, T., Lin, H., Gao, Y., Matyushov, A., Yu, G., Chen, H., et al. (2017). Acoustically Actuated Ultra-Compact Nems Magnetoelectric Antennas. Nat. Commun. 8 (1), 296-298. doi:10.1038/s41467-017-00343-8

Neumaier, D., Pindl, S., and Lemme, M. C. (2019). Integrating Graphene Into Semiconductor Fabrication Lines. Nat. Mater. 18 (6), 525-529. doi:10.1038/ s41563-019-0359-7

Ozger, M., Cetinkaya, O., and Akan, O. B. (2018). Energy Harvesting Cognitive Radio Networking for Iot-Enabled Smart Grid. Mobile Netw. Appl. 23 (4), 956-966. doi:10.1007/s11036-017-0961-3

Pehlivanoglu, E. B. (2018). "Harvesting-Throughput Trade-Off for WirelessPowered Smart Grid Iot Applications: An Experimental Study" in 2018
IEEE International Conference on Communications (ICC) (IEEE), 1-6. doi:10.1109/icc.2018.8422487

Purdie, D. G., Pugno, N. M., Taniguchi, T., Watanabe, K., Ferrari, A. C., and Lombardo, A. (2018). Cleaning Interfaces in Layered Materials Heterostructures. Nat. Commun. 9 (1), 5387-5412. doi:10.1038/s41467-01807558-3

Rodrigo, D., Limaj, O., Janner, D., Etezadi, D., Garcia de Abajo, F. J., Pruneri, V., et al. (2015). Mid-infrared Plasmonic Biosensing With Graphene. Science 349 (6244), 165-168. doi:10.1126/science.aab2051

Romagnoli, M., Sorianello, V., Midrio, M., Koppens, F. H. L., Huyghebaert, C., Neumaier, D., et al. (2018). Graphene-Based Integrated Photonics for NextGeneration Datacom and Telecom. Nat. Rev. Mater. 3 (10), 392-414. doi:10.1038/s41578-018-0040-9

Rong, Z., Leeson, M. S., Higgins, M. D., and Lu, Y. (2018). Nano-Rectenna Powered Body-Centric Nano-Networks in the Terahertz Band. Healthc. Technol. Lett. 5 (4), 113-117. doi:10.1049/htl.2017.0034

Rong, Z., Leeson, M. S., Higgins, M. D., and Lu, Y. (2017). Simultaneous Wireless Information and Power Transfer for Af Relaying Nanonetworks in the Terahertz Band. Nano Commun. Networks. 14, 1-8. doi:10.1016/ j.nancom.2017.08.003

Rutherglen, C., and Burke, P. (2007). Carbon Nanotube Radio. Nano Lett. 7 (11), 3296-3299. doi:10.1021/nl0714839

Rutherglen, C., Jain, D., and Burke, P. (2009). Nanotube Electronics for Radiofrequency Applications. Nat. Nanotech. 4 (12), 811-819. doi:10.1038/ nnano.2009.355

Sarkar, D., Liu, W., Xie, X., Anselmo, A. C., Mitragotri, S., and Banerjee, K. (2014). Mos2 Field-Effect Transistor for Next-Generation Label-Free Biosensors. ACS nano. 8 (4), 3992-4003. doi:10.1021/nn5009148

Schoch, R. B., Han, J., and Renaud, P. (2008). Transport Phenomena in Nanofluidics. Rev. Mod. Phys. 80 (3), 839-883. doi:10.1103/revmodphys.80.839

Schöner, M. G., Simon, R., and Schöner, C. R. (2016). Acoustic Communication in Plant-Animal Interactions. Curr. Opin. Plant Biol. 32, 88-95. doi:10.1016/ j.pbi.2016.06.011

Schroter, M., Claus, M., Sakalas, P., Haferlach, M., and Wang, D. (2013). Carbon Nanotube Fet Technology for Radio-Frequency Electronics: State-Of-The-Art Overview. IEEE J. Electron. Devices Soc. 1 (1), 9-20. doi:10.1109/ jeds.2013.2244641

Sengupta, K., Nagatsuma, T., and Mittleman, D. M. (2018). Terahertz Integrated Electronic and Hybrid Electronic-Photonic Systems. Nat. Electron. 1 (12), 622-635. doi:10.1038/s41928-018-0173-2

Seol, M.-L., Han, J.-W., Park, S.-J., Jeon, S.-B., and Choi, Y.-K. (2016). Hybrid Energy Harvester With Simultaneous Triboelectric and Electromagnetic Generation from an Embedded Floating Oscillator in a Single Package. Nano Energy. 23, 50-59. doi:10.1016/j.nanoen.2016.03.004

Shi, B., Li, Z., and Fan, Y. (2018). Implantable Energy-Harvesting Devices. Adv. Mater. 30 (44), 1801511. doi:10.1002/adma.201801511

Shi, Q. (2019). Bioactuators Based on Stimulus-Responsive Hydrogels and Their Emerging Biomedical Applications. NPG Asia Mater. 11 (1), 1-21. doi:10.1038/ s41427-019-0165-3

Singh, P. K. (2016). "Graphene-Based Plasmonic Phase Modulator for TerahertzBand Communication," in 2016 10th European Conference on Antennas and Propagation (EuCAP) (IEEE), 1-5. doi:10.1109/eucap.2016.7481218

Singh, S. P., Singh, S., Guo, W., Mishra, S., and Kumar, S. (2020). Radiation Absorption Noise for Molecular Information Transfer. IEEE Access. 8, 6379-6387. doi:10.1109/access.2019.2963485

Sliper, S. T., Cetinkaya, O., Weddell, A. S., Al-Hashimi, B., and Merrett, G. V. (2020). Energy-Driven Computing. Phil. Trans. R. Soc. A. 378 (2164), 20190158. doi:10.1098/rsta.2019.0158

Song, A., Stojanovic, M., and Chitre, M. (2019). Editorial Underwater Acoustic Communications: Where We Stand and What Is Next? IEEE J. Oceanic Eng. 44 (1), 1. doi:10.1109/joe.2018.2883872

Sun, Z., Martinez, A., and Wang, F. (2016). Optical Modulators With 2d Layered Materials. Nat. Photon. 10 (4), 227-238. doi:10.1038/nphoton.2016.15

Szunerits, S., and Boukherroub, R. (2018). Graphene-Based Biosensors. Interf. Focus. 8 (3), 20160132. doi:10.1098/rsfs.2016.0132

Tekbiyık, K. (2020). Spectrum Sensing and Signal Identification With Deep Learning Based on Spectral Correlation Function. IEEE Trans. Vehicular Technology., 1. doi:10.1109/TVT.2021.3109236 
Tian, H. (2015). A Flexible, Transparent and Ultrathin Single-Layer Graphene Earphone. RSC Adv. 5 (22), 17 366-417 371. doi:10.1039/c4ral6047a

Todorović, D. (2015). Multilayer Graphene Condenser Microphone. 2D Mater. 2 (4), 045013. doi:10.1088/2053-1583/2/4/045013

Tsang, D. K. H. (2019). Chemically Functionalised Graphene Fet Biosensor for the Label-Free Sensing of Exosomes. Scientific Rep. 9 (1), 1-10. doi:10.1038/s41598019-50412-9

Tu, T., Ju, Z.-Y., Li, Y.-T., Gou, G.-Y., Tian, Y., Wu, F., et al. (2019). A Novel Thermal Acoustic Device Based on Vertical Graphene Film. AIP Adv. 9 (7), 075302. doi:10.1063/1.5096220

Vahala, K. J. (2003). Optical Microcavities. Nature. 424 (6950), 839-846. doi:10.1038/nature01939

VanArsdale, E., Pitzer, J., Payne, G. F., and Bentley, W. E. (2020). Redox Electrochemistry to Interrogate and Control Biomolecular Communication. Iscience. 23 (9), 101545. doi:10.1016/j.isci.2020.101545

Wagih, M. (2020). Real-world Performance of Sub-1 Ghz and 2.4 Ghz Textile Antennas for Rf-Powered Body Area Networks. IEEE Access. 8, 133 746-133 756. doi:10.1109/access.2020.3011603

Walker, M. I., Ubych, K., Saraswat, V., Chalklen, E. A., Braeuninger-Weimer, P., Caneva, S., et al. (2017). Extrinsic Cation Selectivity of $2 \mathrm{~d}$ Membranes. ACS nano. 11 (2), 1340-1346. doi:10.1021/acsnano.6b06034

Wang, Z. L., and Wu, W. (2012). Nanotechnology-Enabled Energy Harvesting for Self-Powered Micro-/Nanosystems. Angew. Chem. Int. Ed. Engl. 51 (47), 11700-11721. doi:10.1002/anie.201201656

Weddell, A. S., Magno, M., Merrett, G. V., Brunelli, D., Bashir, M., and Benini, L. (2013). "A Survey of Multi-Source Energy Harvesting Systems," in 2013 Design, Automation \& Test in Europe Conference \& Exhibition (DATE) (IEEE), 905-908. doi:10.7873/DATE.2013.190

Wei-Yu Chiu, H., Jiang, J., Nallanathan, A., and Poor, H. V. (2012). Wideband Spectrum Sensing With Sub-Nyquist Sampling in Cognitive Radios. IEEE Trans. Signal. Process. 60 (11), 6068-6073. doi:10.1109/ tsp. 2012.2212892

Xu, Q., Schmidt, B., Pradhan, S., and Lipson, M. (2005). Micrometre-Scale Silicon Electro-Optic Modulator. Nature. 435 (7040), 325-327. doi:10.1038/ nature 03569

Xu, S. C., Man, B. Y., Jiang, S. Z., Chen, C. S., Yang, C., Liu, M., et al. (2013). Flexible and Transparent Graphene-Based Loudspeakers. Appl. Phys. Lett. 102 (15), 151902. doi:10.1063/1.4802079

Xu, Z., Dong, X., and Bornemann, J. (2014). Design of a Reconfigurable Mimo System for Thz Communications Based on Graphene Antennas. IEEE Trans. Thz Sci. Technol. 4 (5), 609-617. doi:10.1109/ thz.2014.2331496

Yang, K., Bi, D., Deng, Y., Zhang, R., Rahman, M. M. U., Ali, N. A., et al. (2020). A Comprehensive Survey on Hybrid Communication in Context of Molecular Communication and Terahertz Communication for Body-Centric Nanonetworks. IEEE Trans. Mol. Biol. Multi-Scale Commun. 6 (2), 107-133. doi:10.1109/tmbmc.2020.3017146
Yu, S., Wu, X., Wang, Y., Guo, X., and Tong, L. (2017). 2d Materials for Optical Modulation: Challenges and Opportunities. Adv. Mater. 29 (14), 1606128. doi:10.1002/adma.201606128

Yuan, W., and Shi, G. (2013). Graphene-Based Gas Sensors. J. Mater. Chem. A. 1 (35), 10 078-110 091. doi:10.1039/c3ta11774j

Yucek, T., and Arslan, H. (2009). A Survey of Spectrum Sensing Algorithms for Cognitive Radio Applications. IEEE Commun. Surv. Tutorials. 11 (1), 116-130. doi:10.1109/surv.2009.090109

Zalonis, A., and Polydoros, A. (2005). "Flexible-Radio: A General Framework With Phy-Layer Algorithm-Design Insights," in EUROCON 2005-The International Conference on" Computer as a Tool (IEEE), 120-123.

Zhao, Z. (2020). "An Energy Efficiency Multi-Level Transmission Strategy Based on Underwater Multimodal Communication in Uwsns," in IEEE INFOCOM 2020-IEEE Conference on Computer Communications (IEEE), 1579-1587. doi:10.1109/infocom41043.2020.9155381

Zheng, Q., Shi, B., Fan, F., Wang, X., Yan, L., Yuan, W., et al. (2014). In Vivo powering of Pacemaker by Breathing-Driven Implanted Triboelectric Nanogenerator. Adv. Mater. 26 (33), 5851-5856. doi:10.1002/adma.201402064

Zheng, S., Chen, S., Qi, P., Zhou, H., and Yang, X. (2020). Spectrum Sensing Based on Deep Learning Classification for Cognitive Radios. China Commun. 17 (2), 138-148. doi:10.23919/jcc.2020.02.012

Zhou, Q., and Zettl, A. (2013). Electrostatic Graphene Loudspeaker. Appl. Phys. Lett. 102 (22), 223109. doi:10.1063/1.4806974

Zhou, Q., Zheng, J., Onishi, S., Crommie, M. F., and Zettl, A. K. (2015). Graphene Electrostatic Microphone and Ultrasonic Radio. Proc. Natl. Acad. Sci. USA. 112 (29), 8942-8946. doi:10.1073/pnas.1505800112

Zhou, Y., Zhang, M., Guo, Z., Miao, L., Han, S.-T., Wang, Z., et al. (2017). Recent Advances in Black Phosphorus-Based Photonics, Electronics, Sensors and Energy Devices. Mater. Horiz. 4 (6), 997-1019. doi:10.1039/c7mh00543a

Conflict of Interest: The authors declare that the research was conducted in the absence of any commercial or financial relationships that could be construed as a potential conflict of interest.

Publisher's Note: All claims expressed in this article are solely those of the authors and do not necessarily represent those of their affiliated organizations, or those of the publisher, the editors and the reviewers. Any product that may be evaluated in this article, or claim that may be made by its manufacturer, is not guaranteed or endorsed by the publisher.

Copyright $\odot 2021$ Civas, Cetinkaya, Kuscu and Akan. This is an open-access article distributed under the terms of the Creative Commons Attribution License (CC BY). The use, distribution or reproduction in other forums is permitted, provided the original author(s) and the copyright owner(s) are credited and that the original publication in this journal is cited, in accordance with accepted academic practice. No use, distribution or reproduction is permitted which does not comply with these terms. 